EDINBURGH-97/11

FTUV/99-4

IFIC/99-4

ROME1-1181/97

ROM $2 \mathrm{~F} / 99 / 04$

February 1, 2008

\title{
Non-Perturbative Renormalization of Lattice Four-Fermion Operators without Power Subtractions
}

\author{
A. Donini ${ }^{a}$, V. Giménez ${ }^{b}$, G. Martinelli ${ }^{a}$, M. Talevi ${ }^{c}$, A. Vladikas ${ }^{d}$ \\ ${ }^{a}$ Dip. di Fisica, Univ. di Roma "La Sapienza" and INFN, Sezione di Roma, \\ P.le A. Moro 2, I-00185 Roma, Italy. \\ ${ }^{b}$ Dep. de Fisica Teorica and IFIC, Univ. de Valencia, \\ Dr. Moliner 50, E-46100, Burjassot, Valencia, Spain \\ ${ }^{c}$ Department of Physics \& Astronomy, University of Edinburgh \\ The King's Buildings, Edinburgh EH9 3JZ, UK \\ ${ }^{d}$ INFN, Sezione di Roma II, and Dip. di Fisica, Univ. di Roma "Tor Vergata", \\ Via della Ricerca Scientifica 1, I-00133 Roma, Italy.
}

\begin{abstract}
A general non-perturbative analysis of the renormalization properties of $\Delta I=3 / 2$ four-fermion operators in the framework of lattice regularization with Wilson fermions is presented. We discuss the non-perturbative determination of the operator renormalization constants in the lattice Regularization Independent (RI or MOM) scheme. We also discuss the determination of the finite lattice subtraction coefficients from Ward Identities. We prove that, at large external virtualities, the determination of the lattice mixing coefficients, obtained using the RI renormalization scheme, is equivalent to that based on Ward Identities, in the continuum and chiral limits. As a feasibility study of our method, we compute the mixing matrix at several renormalization scales, for three values of the lattice coupling $\beta$, using the Wilson and tree-level improved SW-Clover actions.
\end{abstract}




\section{Introduction}

The renormalization constants of lattice operators are necessary ingredients in the prediction of physical amplitudes from lattice matrix elements. Schematically, the physical amplitude $A_{\alpha \rightarrow \beta}$ associated to the transition $\alpha \rightarrow \beta$ induced by the composite operator $O$ is given, in the formalism of the Operator Product Expansion (OPE), by

$$
A_{\alpha \rightarrow \beta}=C_{W}(\mu) Z_{O}(a \mu)\langle\alpha|O(a)| \beta\rangle
$$

where $C_{W}$ is the Wilson coefficient, $\mu$ is the renormalization scale, $a$ is the lattice spacing, $\langle\alpha|O(a)| \beta\rangle$ is the matrix element of the bare lattice operator relevant to the physical process and $Z_{O}$ its renormalization constant is calculated in perturbation theory at the renormalization scale $\mu$. It depends on the renormalization scheme (a mass-independent scheme is implied throughout this paper). Wilson coefficients are known to NLO; see refs. [1]-[5]. The matrix element must be calculated non-perturbatively; the only known method for computing it from first principles (at a fixed cutoff $a^{-1}$ ) is lattice QCD [6]. The operator renormalization constant $Z_{O}$ is the link between the matrix element, regularized on the lattice, and its renormalized, continuum counterpart. So far, three methods have been implemented to calculate it:

1. Lattice Perturbation Theory (PT) [7]- 9].

2. Lattice Ward Identities (WI), in the spirit of ref. [10, 11], either with hadronic states (see [12]-[17]), or with quark states (see [14, 15] and, more recently [18]), or even with the Schrödinger functional (see [19]). The WI method is nonperturbative.

3. The Non-Perturbative (NP) method of [20], which consists in imposing renormalization conditions at quark correlation functions with operator insertions, at a given scale (see [20]-24]).

Once the systematic errors of these calculations are understood and kept under control, the matrix elements, computed in simulations, can be properly renormalized and reliable non-perturbative estimates of physical quantities can be obtained.

The simplest case is that of the matrix elements of two-fermion (dimension-three) operators, from which, for example, the decay constants of light vector and pseudoscalar mesons can be extracted. Several complications are avoided due to the fact that the operators renormalize multiplicatively. The situation is less straightforward in the case of four-fermion (dimension-six) operators. Their renormalization is also additive; mixing occurs with other operators of the same dimension and, in some cases, also with operators of lower dimensionality. These operators must then be subtracted in order to make the original operator finite. Mixing with lower-dimensional operators

\footnotetext{
${ }^{1}$ Here our notation is schematic: in general we have a set of operators and $Z_{O}$ is a matrix which mixes those operators which form a complete basis under renormalization.
} 
(e.g in the problem of understanding the $\Delta I=1 / 2$ rule) is characterized by several theoretical and technical subtleties, which have been addressed in [25].

This paper consists in a study of the renormalization properties of the complete basis of dimension-six four-fermion operators in the absence of power subtractions (i.e. operators mixing only with others of equal dimension), using non-perturbative methods. We will discuss below several phenomenological applications of this problem, related to light (strange) flavour phenomenology. We point out however, that since we are dealing with a mass-independent renormalization scheme, our results can also be applied to heavy-flavour phenomenology (e.g. the $\Delta B=2$ process). This calculation is also of relevance to the evaluation of the $\Delta I=1 / 2$ matrix elements, since the anomalous dimension of the octet operators is unchanged by the mixing with lower dimensional operators (and the related power subtractions) [26]. The most common examples, concerning $K$-meson decays, which involve operator mixing without power subtractions are the following (note that $\mathrm{SU}(2)$-isospin breaking effects are neglected):

- The study of $K^{0}-\bar{K}^{0}$ oscillations involves the computation of $B_{K}$ (the B-parameter of the $K$-meson), which is obtained from the $\Delta S=2$ matrix element $\left\langle\bar{K}^{0}\left|O^{\Delta S=2}\right| K^{0}\right\rangle$ of the operator

$$
O^{\Delta S=2}=\left(\bar{s} \gamma_{\mu}^{L} d\right)\left(\bar{s} \gamma_{\mu}^{L} d\right)
$$

where $s$ and $d$ stand for strange and down quarks. Our conventions for Dirac and colour matrices, indices etc. are given in Appendix A.

- The study of the $\Delta I=3 / 2$ sector of the decay $K \rightarrow \pi \pi$ involves the computation of the matrix elements $\left\langle\pi \pi\left|O_{9,10}^{3 / 2}\right| K\right\rangle$ of the operators

$$
\begin{aligned}
& O_{9}^{3 / 2}=\left(\bar{s}^{A} \gamma_{\mu}^{L} d^{A}\right)\left(\bar{u}^{B} \gamma_{\mu}^{L} u^{B}\right)+\left(\bar{s}^{A} \gamma_{\mu}^{L} u^{A}\right)\left(\bar{u}^{B} \gamma_{\mu}^{L} d^{B}\right)-\left(\bar{s}^{A} \gamma_{\mu}^{L} d^{A}\right)\left(\bar{d}^{B} \gamma_{\mu}^{L} d^{B}\right) \\
& O_{10}^{3 / 2}=\left(\bar{s}^{A} \gamma_{\mu}^{L} d^{B}\right)\left(\bar{u}^{B} \gamma_{\mu}^{L} u^{A}\right)+\left(\bar{s}^{A} \gamma_{\mu}^{L} u^{B}\right)\left(\bar{u}^{B} \gamma_{\mu}^{L} d^{A}\right)-\left(\bar{s}^{A} \gamma_{\mu}^{L} d^{B}\right)\left(\bar{d}^{B} \gamma_{\mu}^{L} d^{A}\right)
\end{aligned}
$$

with $u$ standing for the up quark and $A, B$ denoting colour indices. The above operators transform as members of the $(27,1)$ representation of the $S U(3)_{L} \otimes$ $S U(3)_{R}$ chiral group. Moreover, the study of the electropenguin contribution to the decay $K \rightarrow \pi \pi$ involves the computation of the matrix element $\left\langle\pi \pi\left|O_{7,8}^{3 / 2}\right| K\right\rangle$ of the operators

$$
\begin{aligned}
& O_{7}^{3 / 2}=\left(\bar{s}^{A} \gamma_{\mu}^{L} d^{A}\right)\left(\bar{u}^{B} \gamma_{\mu}^{R} u^{B}\right)+\left(\bar{s}^{A} \gamma_{\mu}^{L} u^{A}\right)\left(\bar{u}^{B} \gamma_{\mu}^{R} d^{B}\right)-\left(\bar{s}^{A} \gamma_{\mu}^{L} d^{A}\right)\left(\bar{d}^{B} \gamma_{\mu}^{R} d^{B}\right) \\
& O_{8}^{3 / 2}=\left(\bar{s}^{A} \gamma_{\mu}^{L} d^{B}\right)\left(\bar{u}^{B} \gamma_{\mu}^{R} u^{A}\right)+\left(\bar{s}^{A} \gamma_{\mu}^{L} u^{B}\right)\left(\bar{u}^{B} \gamma_{\mu}^{R} d^{A}\right)-\left(\bar{s}^{A} \gamma_{\mu}^{L} d^{B}\right)\left(\bar{d}^{B} \gamma_{\mu}^{R} d^{A}\right)
\end{aligned}
$$

which are also $\Delta I=3 / 2$, but transform as an $(8,8)$ representation of the chiral group. These $K \rightarrow \pi \pi$ matrix elements are essential to the calculation of $\epsilon^{\prime} / \epsilon$. They can be obtained, through soft pion theorems, from the matrix elements $\left\langle\pi^{+}\left|O_{k}^{3 / 2}\right| K^{+}\right\rangle$(for $k=7,8,9,10$ ). From the single-state matrix elements, the corresponding $B$-parameters, $B_{7,8}^{3 / 2}$, can be extracted. Note that, in the limit of 
degenerate quark masses, both operators $O_{9,10}^{3 / 2}$, having a "left-left" Dirac structure, renormalize in the same way as the $O^{\Delta S=2}$ operator. Moreover, the matrix elements $\left\langle\pi^{+}\left|O_{9,10}^{3 / 2}\right| K^{+}\right\rangle$have the same $B$-parameter as $\left\langle\bar{K}^{0}\left|O^{\Delta S=2}\right| K^{0}\right\rangle$. These matrix elements should vanish in the chiral limit. On the contrary, the operators $O_{7,8}^{3 / 2}$, having a "left -right" Dirac structure, obey different renormalization properties. The corresponding matrix elements $\left\langle\pi^{+}\left|O_{7,8}^{3 / 2}\right| K^{+}\right\rangle$do not vanish in the chiral limit

- Important information on the physics beyond the Standard Model, such as SUSY, can be obtained by studying FCNC processes and, in particular, $\Delta F=2$ transitions (see [27] and references therein for a discussion). Besides the $B$-parameters of the operators listed above, such processes also require the knowledge of the $B$-parameters of the operators

$$
\begin{aligned}
& \left(\bar{s}^{A}\left(1-\gamma_{5}\right) d^{A}\right)\left(\bar{s}^{B}\left(1-\gamma_{5}\right) d^{B}\right) \\
& \left(\bar{s}^{A}\left(1-\gamma_{5}\right) d^{B}\right)\left(\bar{s}^{B}\left(1-\gamma_{5}\right) d^{A}\right) \\
& \left(\bar{s}^{A}\left(1-\gamma_{5}\right) d^{A}\right)\left(\bar{s}^{B}\left(1+\gamma_{5}\right) d^{B}\right) \\
& \left(\bar{s}^{A}\left(1-\gamma_{5}\right) d^{B}\right)\left(\bar{s}^{B}\left(1+\gamma_{5}\right) d^{A}\right)
\end{aligned}
$$

Recent lattice results on all these $B$-parameters (with Wilson fermions) can be found in refs. [18, 28, 29, 30].

The matrix elements discussed above are extracted from the large time asymptotic behaviour of three-point correlation functions of the form $\left\langle P_{K} O^{\Delta S=2} P_{K}\right\rangle$ and $\left\langle P_{K}^{\dagger} O_{k}^{3 / 2} P_{\pi}\right\rangle$ (for $k=7,8,9,10$ ) where $P_{K}$ and $P_{\pi}$ are pseudoscalar sources of suitable quark flavour. Expressed in terms of traces of quark propagators, these correlation functions correspond to the so-called "eight"- shaped quark diagrams. Any "eye"shaped contributions of these correlation functions cancel in the limit of degenerate up and down quarks, which is the case under study.

The paper is organized as follows: in sec. 目 we illustrate, as a concrete example of the problems arising in lattice renormalization, the mixing of the operator $O^{\Delta S=2}$. Its matrix element $\left\langle\bar{K}^{0}\left|O^{\Delta S=2}\right| K^{0}\right\rangle$ is very sensitive to the various systematic errors which affect its chiral behaviour and therefore provides a good case-study. In sec. 3 we discuss in full detail the problem of mixing under renormalization of all four-fermion dimensionsix operators. The operators are classified according to their discrete symmetries and an operator basis, convenient for our purposes, is chosen. Subsequently, the operator mixing under renormalization is divided into two parts: mixing that would occur even if all the symmetries were respected by the regulator, and mixing which is induced on the lattice by the chiral symmetry breaking of the Wilson term. In sec. 1 the NP renormalization is applied to the cases at hand. The renormalization conditions of the so-called RI (or MOM) scheme are worked out in terms of projected amputated Green functions, for the complete operator basist. In sec. 5 we derive WI's suitable for the

\footnotetext{
${ }^{2}$ For continuum calculations in the RI scheme and the anomalous dimensions of the complete basis of operators, calculated in PT to NLO, see ref. [5].
} 
determination of the lattice mixing coefficients in the chiral limit. We show that, in this respect, the RI and the WI methods are equivalent in the region of large external momenta. We also show the independence of the lattice mixing coefficients from the renormalization scale. Finally, in sec. 6 we present the renormalization constants and lattice mixing coefficients, obtained with the NP method, with both the Wilson and the tree-level improved Clover action at several values of the lattice bare coupling $\beta$. Some of these results have been used in refs. [29, 30] in order to derive fully non-perturbative estimates of the $B$-parameters.

\section{Renormalization of $O^{\Delta S=2}$ and systematic er- rors}

The testing ground for the restoration of chiral symmetry in the continuum limit of lattice computations has been the chiral behaviour of the matrix element $\left\langle\bar{K}^{0}\left|O^{\Delta S=2}\right| K^{0}\right\rangle$, which, if properly renormalized, vanishes when the $K$-meson becomes massless [31]. Although early attempts with Wilson fermions [32]-[34] have given reasonable measurements of $B_{K}$, they have been less successful in controlling this chiral behaviour? The root of the problem is the operator subtraction outlined above, which we now discuss in some detail: $O^{\Delta S=2}$ mixes with other operators $O_{i}$ of the same dimension but with "wrong naive chirality". Thus, the ( $\mu$-dependent) $K^{0}-\bar{K}^{0}$ matrix element of the renormalized operator $\hat{O}^{\Delta S=2}$ is given in terms of the (a-dependent) bare matrix elements by:

$$
\left\langle\bar{K}^{0}\left|\hat{O}^{\Delta S=2}(\mu)\right| K^{0}\right\rangle=\lim _{a \rightarrow 0}\left\langle\bar{K}^{0}\left|Z_{0}^{\Delta S=2}\left(a \mu, g_{0}^{2}\right)\left[O^{\Delta S=2}(a)+\sum_{i} Z_{i}^{\Delta S=2}\left(g_{0}^{2}\right) O_{i}(a)\right]\right| K^{0}\right\rangle
$$

The overall renormalization constant $Z_{0}^{\Delta S=2}\left(a \mu, g_{0}^{2}\right)$ is logarithmically divergent whereas the $Z_{i}^{\Delta S=2}\left(g_{0}^{2}\right)$ 's are finite mixing coefficients; $g_{0}^{2}(a)$ is the lattice bare coupling (also expressed as $\left.\beta=6 / g_{0}^{2}\right)$. All $Z$ 's can be calculated, at least in principle, in perturbation theory (PT), provided that $\mu, a^{-1} \gg \Lambda_{\mathrm{QCD}}$. The one-loop perturbative calculation of the $Z$ 's, both for the Wilson and Clover lattice actions, has been performed in [8]- [9]. Although the renormalized operator $\hat{O}^{\Delta S=2}(\mu)$ is constructed so as to have the correct chiral properties in the continuum limit, the implementation of operator mixing in early works [32]-34] was subject to two main systematic errors:

1. The overall renormalization constant $Z_{0}^{\Delta S=2}$ and the mixing coefficients $Z_{i}^{\Delta S=2}$ were calculated in one-loop PT. Thus, they suffered from $\mathcal{O}\left(g_{0}^{4}\right)$ systematic errors.

\footnotetext{
${ }^{3} B_{K}$ has also been obtained with staggered fermions 35 mainly in the quenched approximation (see ref. [36] for a review). The (surviving) chiral symmetry in the staggered fermion formalism ensures the vanishing of the relevant matrix element in the chiral limit.
} 
2. The bare matrix elements $\left\langle\bar{K}^{0}\left|O^{\Delta S=2}(a)\right| K^{0}\right\rangle$ and $\left\langle\bar{K}^{0}\left|O_{i}(a)\right| K^{0}\right\rangle$ were calculated non-perturbatively (numerically) with the Wilson action at fixed cutoff $a$ (i.e. fixed coupling $\left.g_{0}^{2}\right)$. They were therefore subject to $\mathcal{O}(a)$ systematic errors.

Both sources of systematic error may be considered responsible for the non-vanishing of the matrix element $\left\langle\bar{K}^{0}\left|\hat{O}^{\Delta S=2}\right| K^{0}\right\rangle$ in the chiral limit. In order to reduce these errors, the following remedies have been proposed:

1. Boosted Perturbation Theory (BPT) should, according to [37], improve the behaviour of the perturbative series of the renormalization constants. The systematic error due to the 1-loop truncation of the BPT estimates of the $Z$ 's remains $\mathcal{O}\left(g_{0}^{4}\right)$, but it is hoped that it is smaller than the $\mathcal{O}\left(g_{0}^{4}\right)$ error of the original PT expansion.

2. Discretization errors can be reduced by using improved actions and operators. To this purpose, besides the Wilson action, the Clover action [38 has also been implemented in the calculation of the weak matrix elements $\left\langle\overline{K^{0}}\left|O^{\Delta S=2}(a)\right| K^{0}\right\rangle$ and $\left\langle\bar{K}^{0}\left|O_{i}(a)\right| K^{0}\right\rangle$. Matrix elements of tree-level improved operators, calculated with the tree-level improved Clover action, have discretization errors of $\mathcal{O}\left(g_{0}^{2} a\right)$, instead of $\mathcal{O}(a)$ as in the Wilson caset.

3. Using the non-perturbative (NP) method of [20, 21], a more accurate evaluation of the renormalization constants can be achieved. The method consists in imposing renormalization conditions directly on quark four-point Green functions with operator insertions. Higher-order contributions, including non-perturbative effects, are taken into account. With this method, the $Z$ 's have the same discretization errors (due to the finiteness of the lattice spacing) as the operator matrix elements we are ultimately interested in. Another non-perturbative method consists in the determination of the mixing coefficients from WI's on quark states [18]. We stress that the WI method can only be applied to the lattice mixing coefficients; the overall renormalization $Z_{0}^{\Delta S=2}\left(a \mu, g_{0}^{2}\right)$ can only be determined non-perturbatively from NP methods; e.g. those of refs. [19, 20, 21]. Both WI and NP results are expected to have similar discretization errors.

The systematic errors of the operator renormalization described by eq. (6) depend upon the method of calculation of its various terms: if PT (or BPT) is used for the $Z$ 's and the Wilson action for the matrix elements, the renormalization is good to $\mathcal{O}\left(g_{0}^{4}\right)$ and $\mathcal{O}(a)$. If PT (or BPT) is used for the Z's and the tree-level Clover action is used for the matrix elements, the renormalization is good to $\mathcal{O}\left(g_{0}^{4}\right)$ and $\mathcal{O}\left(g_{0}^{2} a\right)$. If NP (or WI) renormalization is used for the $Z$ 's then the error is $\mathcal{O}(a)$ or $\mathcal{O}\left(g_{0}^{2} a\right)$, depending on the action used (Wilson or tree-level Clover). Of course, the same considerations are valid for the renormalization of the operators of eqs. (3)-(5).

${ }^{4}$ Recently, it was shown that the non-perturbative Clover improvement, proposed in 19 reduces the above error to $\mathcal{O}\left(a^{2}\right)$. We have not implemented this version of the Clover action in the present work, because it involves more complicated operator mixing. 
A study of the effect of each of these three improvements had first been made in ref. [39], in which a direct comparison of the various errors in the computation of the $\Delta S=2$ matrix element was carried out. The main result of [39] is that the chiral behaviour of the renormalized $\left\langle\bar{K}^{0}\left|\hat{O}^{\Delta S=2}\right| K^{0}\right\rangle$ is not improved significantly if BPT is used for the calculation of the mixing constants $Z_{i}^{\Delta S=2}$ and the Clover action is implemented in the computation of the matrix elements \%. What really makes a difference is the NP computation of the $Z$ 's, which was shown to improve significantly the chiral behaviour of the matrix element. This conclusion has been confirmed in [18] with Wilson fermions and for several $\beta$ values. We stress that in [18] the nonperturbative computation of the mixing $Z$ 's was performed using WI's on quark states, as opposed to the NP method of [39].

In all early works, ref. [39] included, the renormalization of $O^{\Delta S=2}$ consisted in the mixing with only three dimension-six operators with the "wrong naive chirality". This mixing had been explicitly derived in [8], using lattice PT at one-loop. However, by applying the general symmetry arguments of 41] to the case in hand, one can show that a fourth operator should be added in the sum of eq. (6), as pointed out in refs. 18, 24, 33]. This operator, absent at the one-loop level of PT, has a mixing constant which is at least $\mathcal{O}\left(g_{0}^{4}\right)$. It gives a small (but non-negligible) contribution to the restoration of the chiral behaviour of the matrix element [24]. In refs. [24, 29] and the present work, this operator, omitted in ref. [39], has been properly taken into account.

\section{Mixing of dimension-six four-fermion opera- tors on the lattice}

In this section, we introduce the basic notation and study the mixing of generic fourfermion operators in the presence of explicit chiral symmetry breaking, induced by the Wilson term. This implies that mixing with operators with the "wrong naive chirality" is allowed, even in the chiral limit. Following [41] (see also [33]), we obtain the complete basis of dimension-six, four-fermion operators which mix under renormalization, relying on general symmetry arguments based on the vector flavour symmetry, which survives on the lattice. In order to solve this problem, it is convenient to work with massless fermions with four distinct flavours $\psi_{f}, f=1, \ldots, 4$. The correlation functions arising from these operators do not contain "eye"-shaped contributions and therefore their renormalization properties are identical, up to trivial factors, to those of the physical $\Delta S=2$ and $\Delta I=3 / 2$ operators considered in sect. 1 (recall that we are assuming $\mathrm{SU}(2)$-isospin symmetry). The introduction of "light" masses (i.e. masses which are much smaller than the renormalization scale) will be discussed in subsect. 5.2. We will not address the problem of "heavy" masses (of the order of, or greater than the typical scales and the UV cutoff) in this work.

${ }^{5}$ For a different conclusion see ref. 40] 


\begin{tabular}{|r|r|r|r|r|r|}
\hline$O_{\Gamma(1)}(2)$ & $\mathcal{P}$ & $\mathcal{C S}^{\prime}$ & $\mathcal{C S}^{\prime \prime}$ & $\mathcal{C P S}^{\prime}$ & $\mathcal{C P S}^{\prime \prime}$ \\
\hline \hline$O_{V V}$ & +1 & +1 & +1 & +1 & +1 \\
$O_{A A}$ & +1 & +1 & +1 & +1 & +1 \\
$O_{P P}$ & +1 & +1 & +1 & +1 & +1 \\
$O_{S S}$ & +1 & +1 & +1 & +1 & +1 \\
$O_{T T}$ & +1 & +1 & +1 & +1 & +1 \\
\hline$O_{V A}$ & -1 & -1 & $-O_{A V}$ & +1 & $O_{A V}$ \\
$O_{A V}$ & -1 & -1 & $-O_{V A}$ & +1 & $O_{V A}$ \\
$O_{S P}$ & -1 & +1 & $O_{P S}$ & -1 & $-O_{P S}$ \\
$O_{P S}$ & -1 & +1 & $O_{S P}$ & -1 & $-O_{S P}$ \\
$O_{T \tilde{T}}$ & -1 & +1 & +1 & -1 & -1 \\
\hline$O_{[V A+A V]}$ & -1 & -1 & -1 & +1 & +1 \\
$O_{[V A-A V]}$ & -1 & -1 & +1 & +1 & -1 \\
$O_{[S P-P S]}$ & -1 & +1 & -1 & -1 & +1 \\
$O_{[S P+P S]}$ & -1 & +1 & +1 & -1 & -1 \\
$O_{T \tilde{T}}$ & -1 & +1 & +1 & -1 & -1 \\
\hline
\end{tabular}

Table 1: Classification of four- fermion operators $O_{\Gamma^{(1)} \Gamma^{(2)}}$ according to $\mathcal{P}$ and useful products of their discrete symmetries $\mathcal{C}, \mathcal{S}^{\prime}$ and $\mathcal{S}^{\prime \prime}$. These properties are also valid for the operators $O_{t^{a} \Gamma^{(1)} t^{a} \Gamma^{(2)}}$. For the operators $O_{\Gamma^{(1)} \Gamma^{(2)}}^{F}$ and $O_{t^{a} \Gamma^{(1)} t^{a} \Gamma^{(2)}}^{F}$, we must exchange the entries of the columns $\mathcal{C S}^{\prime} \leftrightarrow \mathcal{C S}^{\prime \prime}$ and $\mathcal{C P} \mathcal{S}^{\prime} \leftrightarrow \mathcal{C P} \mathcal{S}^{\prime \prime}$. Note that $O_{\tilde{T} \tilde{T}}=O_{T T}$ and $O_{T \tilde{T}}=O_{\tilde{T} T}$.

We define the generic four fermion operators

$$
\begin{aligned}
O_{\Gamma^{(1)} \Gamma^{(2)}} & =\left(\bar{\psi}_{1} \Gamma^{(1)} \psi_{2}\right)\left(\bar{\psi}_{3} \Gamma^{(2)} \psi_{4}\right) \\
O_{t^{a} \Gamma^{(1)} t^{a} \Gamma^{(2)}} & =\left(\bar{\psi}_{1} t^{a} \Gamma^{(1)} \psi_{2}\right)\left(\bar{\psi}_{3} t^{a} \Gamma^{(2)} \psi_{4}\right) \\
O_{\Gamma^{(1)} \Gamma^{(2)}}^{F} & =\left(\bar{\psi}_{1} \Gamma^{(1)} \psi_{4}\right)\left(\bar{\psi}_{3} \Gamma^{(2)} \psi_{2}\right) \\
O_{t^{a} \Gamma^{(1)} t^{a} \Gamma^{(2)}}^{F} & =\left(\bar{\psi}_{1} t^{a} \Gamma^{(1)} \psi_{4}\right)\left(\bar{\psi}_{3} t^{a} \Gamma^{(2)} \psi_{2}\right)
\end{aligned}
$$

where $\Gamma^{(1)}$ and $\Gamma^{(2)}$ denote any Dirac matrices and $t^{a}$ are the colour-group generators (referred to as colour matrices in this work). For notation and conventions, see Appendix A.

\subsection{Operator classification according to discrete symme- tries}

Let us start by addressing the problem of mixing of the generic operators of eq. (7) under renormalization. First of all we stress that they cannot mix with operators of lower dimensionality, because such operators do not have the four-flavour content of the 
original ones 5 . Thus, $O_{\Gamma^{(1)} \Gamma^{(2)}}$ can mix with any other dimension-six operator, provided it has the same quantum numbers (i.e. with any operator which has the symmetries of $O_{\Gamma^{(1)} \Gamma^{(2)}}$ and of the action). The generic QCD Wilson lattice action with 4 degenerate quarks is symmetric under parity $\mathcal{P}$, and charge conjugation $\mathcal{C}$. Moreover, there are three other useful (flavour) symmetries of the action, namely the flavour exchange symmetry $\mathcal{S} \equiv\left(\psi_{2} \leftrightarrow \psi_{4}\right)$ and the switching symmetries $\mathcal{S}^{\prime} \equiv\left(\psi_{1} \leftrightarrow \psi_{2}, \psi_{3} \leftrightarrow \psi_{4}\right)$ and $\mathcal{S}^{\prime \prime} \equiv\left(\psi_{1} \leftrightarrow \psi_{4}, \psi_{2} \leftrightarrow \psi_{3}\right)$ 41]. In Table 11 we classify the operators $O_{\Gamma^{(1)} \Gamma^{(2)}}$, for all $\Gamma^{(1)}$ and $\Gamma^{(2)}$ combinations of interest, according to the discrete symmetries $\mathcal{P}, \mathcal{C}$, $\mathcal{S}^{\prime}$ and $\mathcal{S}^{\prime \prime}$. Parity violating operators (middle section of the Table), for which $\mathcal{C S}^{\prime \prime}$ is not a symmetry, have been (anti)symmetrized in order to obtain eigenstates of $\mathcal{C S}^{\prime \prime}$ (bottom section of the Table). We adopt the notation

$$
O_{\left[\Gamma^{(1)} \Gamma^{(2)} \pm \Gamma^{(2)} \Gamma^{(1)}\right]}=O_{\Gamma^{(1)} \Gamma^{(2)}} \pm O_{\Gamma^{(2)} \Gamma^{(1)}}
$$

Note that the results of Table 1 apply also to the operators $O_{t^{a} \Gamma^{(1)} t^{a} \Gamma^{(2)}}$ since, upon performing the symmetry transformations, sign differences, resulting from the presence of the colour $t^{a}$ matrix, disappear because the colour matrices appear quadratically. On the other hand, $O_{\Gamma^{(1)} \Gamma^{(2)}}^{F}$ is obtained by applying $\mathcal{S}$ on $O_{\Gamma^{(1)} \Gamma^{(2)}}$. Since $\mathcal{S}$ transforms $\mathcal{S}^{\prime}$ into $\mathcal{S}^{\prime \prime}$, the properties of Table 1 also apply to $O_{\Gamma^{(1)} \Gamma^{(2)}}^{F}$, but with all $\mathcal{S}^{\prime}$ and $\mathcal{S}^{\prime \prime}$ columns exchanged. Again, the operator $O_{t^{a} \Gamma^{(1)} t^{a} \Gamma^{(2)}}^{F}$ has the same properties as $O_{\Gamma^{(1)} \Gamma^{(2)}}^{F}$, since the colour matrix $t^{a}$ appears quadratically.

Our aim is to find complete bases of operators which mix under renormalization. Thus, the first task is the elimination of the operators which are not independent. In particular, given the mixing of $O_{\Gamma^{(1)} \Gamma^{(2)}}$ with all other allowed $O_{\Gamma^{(i)} \Gamma^{(j)}}$ 's and $O_{\Gamma^{(i)} \Gamma^{(j)}}^{F}$ 's, we do not need to consider also the mixing with the $O_{t^{a} \Gamma^{(i)} t^{a} \Gamma^{(j)}}$ 's and $O_{t^{a} \Gamma^{(i)} t^{a} \Gamma^{(j)}}$ 's, since they can be expressed in terms of the $O_{\Gamma^{(i)} \Gamma^{(j)}}$ 's and the $O_{\Gamma^{(i)} \Gamma^{(j)}}^{F}$ 's. This can be seen by applying the standard identity of colour matrices (Fierz transformation of colour indices)

$$
t_{A B}^{a} t_{C D}^{a}=-\frac{1}{2 N_{c}} \delta_{A B} \delta_{C D}+\frac{1}{2} \delta_{A D} \delta_{C B}
$$

on the $t^{a}$ 's of a given operator. For the operator $O_{t^{a} \Gamma^{(i)} t^{a} \Gamma^{(j)}}$ the result has the general form

$$
O_{t^{a} \Gamma^{(i)} t^{a} \Gamma^{(j)}}=-\frac{1}{2 N_{c}} O_{\Gamma^{(i)} \Gamma^{(j)}}+\frac{1}{2} \sum_{n, m} C_{n m} O_{\Gamma^{(n)} \Gamma^{(m)}}^{F}
$$

where the sum runs over all the Dirac matrices obtained by the Fierz transformation of $\Gamma^{(i)} \Gamma^{(j)}$. The factors $C_{n m}$ are the appropriate constants of the Fierz transformation of the Dirac matrices (see Appendix B for details on Fierz transformations in Dirac space). Analogously we can express $O_{t^{a} \Gamma^{(i)} t^{a} \Gamma^{(j)}}^{F}$ in terms of $O_{\Gamma^{(i)} \Gamma^{(j)}}$ and $O_{\Gamma^{(i)} \Gamma^{(j)}}^{F}$. Therefore, in the following, it is adequate to limit ourselves to the mixing of $O_{\Gamma^{(i)} \Gamma^{(j)}}$ 's and $O_{\Gamma^{(i)} \Gamma^{(j)}}^{F}$ 's, which form a complete basis of 20 independent operators (these are the

${ }^{6}$ This statement remains true for the operators of eqs.(2)-(5). The $\Delta I=1 / 2$ operator however, mixes in general with operators of dimension-five and -three. 
5 operators of the top section of Table 1, the 5 operators of the bottom section and their 10 Fierz counterparts).

Furthermore, by classifying the operators according to the discrete symmetries listed in Table 1, the original basis of 20 operators can be further decomposed into smaller independent bases. An immediate decomposition is that into two bases, of 10 operators each, with definite parity $(\mathcal{P}= \pm 1)$. Further decompositions occur upon using the remaining $\mathcal{C P} \mathcal{S}^{\prime}, \mathcal{C P} \mathcal{S}^{\prime \prime}$ and $\mathcal{S}$ symmetries. These we now perform case by case.

\subsection{Parity conserving operators}

All of the parity conserving operators $O_{\Gamma \Gamma}$ are eigenstates of all the discrete symmetries listed above, with eigenvalue +1 . Therefore, each of them can mix with the other four, and also with the five $O_{\Gamma \Gamma}^{F}$ 's; the complete basis consists of 10 operators. We now rotate our basis into a new one, consisting of 10 operators which are also eigenstates of $\mathcal{S}$ with eigenvalues \pm 1 :

$$
O_{\Gamma \Gamma}^{ \pm}=\frac{1}{2}\left[O_{\Gamma \Gamma} \pm O_{\Gamma \Gamma}^{F}\right]=\frac{1}{2}\left[\left(\bar{\psi}_{1} \Gamma \psi_{2}\right)\left(\bar{\psi}_{3} \Gamma \psi_{4}\right) \pm\left(\bar{\psi}_{1} \Gamma \psi_{4}\right)\left(\bar{\psi}_{3} \Gamma \psi_{2}\right)\right]
$$

Clearly, the $5 O_{\Gamma \Gamma}^{+}$'s, corresponding to $\mathcal{S}=+1$, mix only among themselves; the same is true for the $O_{\Gamma \Gamma}^{-}$'s which have $\mathcal{S}=-1$. Thus the original basis of 10 operators has been decomposed into two independent bases of 5 operators each.

In this work, since we are eventually interested in the renormalization of the operators of eqs. (2)-(5), we opt for the basis

$$
\begin{aligned}
Q_{1}^{ \pm} & \equiv O_{[V V+A A]}^{ \pm} \\
Q_{2}^{ \pm} & \equiv O_{[V V-A A]}^{ \pm} \\
Q_{3}^{ \pm} & \equiv O_{[S S-P P]}^{ \pm} \\
Q_{4}^{ \pm} & \equiv O_{[S S+P P]}^{ \pm} \\
Q_{5}^{ \pm} & \equiv O_{T T}^{ \pm}
\end{aligned}
$$

Other choices of basis can be found in [8, 18]. All such bases are simply linear combinations of each other.

\subsection{Parity violating operators}

The parity violating four- fermion operators listed at the bottom section of Table 1 do not all have identical $\mathcal{C} \mathcal{P} \mathcal{S}^{\prime}$ and $\mathcal{C} \mathcal{P} \mathcal{S}^{\prime \prime}$ values. We will now establish their mixing pattern, with the aid of $\mathcal{S}$ symmetry.

The operator $O_{[V A+A V]}$ mixes with $O_{[V A+A V]}^{F}$ only, forming a basis of two operators with $\mathcal{C P S}^{\prime}=\mathcal{C} \mathcal{P} \mathcal{S}^{\prime \prime}=+1$. In analogy to the parity conserving case, we rotate this 
basis into

$$
O_{[V A+A V]}^{ \pm} \equiv \frac{1}{2}\left[O_{[V A+A V]} \pm O_{[V A+A V]}^{F}\right]
$$

Since $O_{[V A+A V]}^{+}$has $\mathcal{S}=+1$ and $O_{[V A+A V]}^{-}$has $\mathcal{S}=-1$, they do not mix with each other; the two operators renormalize multiplicatively. In other words, the original basis of two operators has been decomposed into two bases of one operator each.

The operators $O_{[V A-A V]}$ and $O_{[S P-P S]}^{F}$ mix, since they both have $\mathcal{C} \mathcal{P} \mathcal{S}^{\prime}=+1$ and $\mathcal{C P S}^{\prime \prime}=-1$. Similarly, $O_{[V A-A V]}^{F}$ and $O_{[S P-P S]}$ form another basis, with $\mathcal{C} \mathcal{P} \mathcal{S}^{\prime}=-1$, $\mathcal{C P S}^{\prime \prime}=+1$. It is convenient to combine these two bases into 4 operators:

$$
\begin{aligned}
O_{[V A-A V]}^{ \pm} & \equiv \frac{1}{2}\left[\left(O_{[V A-A V]} \pm O_{[V A-A V]}^{F}\right]\right. \\
O_{[S P-P S]}^{ \pm} & \equiv \frac{1}{2}\left[O_{[S P-P S]} \pm O_{[S P-P S]}^{F}\right]
\end{aligned}
$$

None of these operators have definite $\mathcal{C P \mathcal { S } ^ { \prime }}$ or $\mathcal{C} \mathcal{P} \mathcal{S}^{\prime \prime}$. However, they have definite $\mathcal{S}= \pm 1$. There is mixing between the two $\mathcal{S}=+1$ operators $\left(O_{[V A-A V]}^{+}, O_{[S P-P S]}^{+}\right)$ and also between the two $\mathcal{S}=-1$ operators $\left(O_{[V A-A V]}^{-}, O_{[S P-P S]}^{-}\right)$.

We follow a similar line of reasoning for the operators $O_{[S P+P S]}, O_{[S P+P S]}^{F}, O_{T \tilde{T}}$ and $O_{T \tilde{T}}^{F}$ (all have $\mathcal{C P} \mathcal{S}^{\prime}=\mathcal{C} \mathcal{P} \mathcal{S}^{\prime \prime}=-1$ ). We rotate the basis of these four operators into

$$
\begin{aligned}
O_{[S P+P S]}^{ \pm} & \equiv \frac{1}{2}\left[O_{[S P+P S]} \pm O_{[S P+P S]}^{F}\right] \\
O_{T \tilde{T}}^{ \pm} & \equiv \frac{1}{2}\left[O_{T \tilde{T}} \pm O_{T \tilde{T}}^{F}\right]
\end{aligned}
$$

Thus, once more, the original basis of the four operators of eq.(15) can be decomposed into two bases, of two operators each, with definite $\mathcal{S}= \pm 1$.

For reasons of notational compactness, we will use the following redefinitions:

$$
\begin{aligned}
\mathcal{Q}_{1}^{ \pm} & \equiv O_{[V A+A V]}^{ \pm} \\
\mathcal{Q}_{2}^{ \pm} & \equiv O_{[V A-A V]}^{ \pm} \\
\mathcal{Q}_{3}^{ \pm} & \equiv-O_{[S P-P S]}^{ \pm} \\
\mathcal{Q}_{4}^{ \pm} & \equiv O_{[S P+P S]}^{ \pm} \\
\mathcal{Q}_{5}^{ \pm} & \equiv O_{T \tilde{T}}^{ \pm}
\end{aligned}
$$

\subsection{Operator subtraction and chiral symmetry}

This completes the discussion of the mixing of dimension-six operators on the lattice, in the general case of 4 distinct flavours (degenerate or not). We now summarize the result to keep in mind. For the parity conserving operators, the renormalization is given by

$$
\hat{Q}_{i}^{ \pm}=Z_{i j}^{ \pm} Q_{j}^{ \pm} \quad(i, j=1, \ldots, 5)
$$


whereas for the parity violating ones we have

$$
\hat{\mathcal{Q}}_{i}^{ \pm}=\mathcal{Z}_{i j}^{ \pm} \mathcal{Q}_{j}^{ \pm} \quad(i, j=1, \ldots, 5)
$$

where $\hat{Q}_{i}^{ \pm}$and $\hat{\mathcal{Q}}_{i}^{ \pm}$are the bases of renormalized operators and $Z_{i j}^{ \pm}$and $\mathcal{Z}_{i j}^{ \pm}$are the renormalization matrices (summation over repeated indices is implied).

On the basis of $\mathcal{C P S}$ symmetries, it is important to notice that the matrix $\mathcal{Z}_{i j}^{ \pm}$is a (relatively sparse) block diagonal matrix. We show this explicitly by re-writing the last equation according to the allowed mixing of the operators derived in the previous Subsection:

$$
\left(\begin{array}{c}
\hat{\mathcal{Q}}_{1} \\
\hat{\mathcal{Q}}_{2} \\
\hat{\mathcal{Q}}_{3} \\
\hat{\mathcal{Q}}_{4} \\
\hat{\mathcal{Q}}_{5}
\end{array}\right)^{ \pm}=\left(\begin{array}{rrrrr}
\mathcal{Z}_{11} & 0 & 0 & 0 & 0 \\
0 & \mathcal{Z}_{22} & \mathcal{Z}_{23} & 0 & 0 \\
0 & \mathcal{Z}_{32} & \mathcal{Z}_{33} & 0 & 0 \\
0 & 0 & 0 & \mathcal{Z}_{44} & \mathcal{Z}_{45} \\
0 & 0 & 0 & \mathcal{Z}_{54} & \mathcal{Z}_{55}
\end{array}\right)^{ \pm}\left(\begin{array}{c}
\mathcal{Q}_{1} \\
\mathcal{Q}_{2} \\
\mathcal{Q}_{3} \\
\mathcal{Q}_{4} \\
\mathcal{Q}_{5}
\end{array}\right)^{ \pm}
$$

The abbreviated notation with the \pm superscript of the column vectors and matrix should be transparent to the reader. In conclusion, the lattice does not induce extra subtractions for the parity violating sector $\left(\mathcal{Q}_{k}^{ \pm} ; k=1, \ldots, 5\right)$, since the mixing occurring according to the pattern of eq. (19), is also valid in the $\chi R S$ scheme; see below.

In the case of parity conserving operators, we proceed in a different way. We find it convenient to separate the above operator mixing into two classes: 1) the first is the lattice subtraction, which consists in correcting the operator mixing, induced by the breaking of chiral symmetry due to the Wilson term of the action; 2) the second is the subtraction which survives in the continuum limit. In order to facilitate this separation, let us suppose that there is a regularization scheme which, unlike the lattice, respects chiral symmetry; i.e. the regularized action has no chiral symmetry breaking term (recall we are working at zero quark mass). We will call this hypothetical scheme the Chirally-symmetric Regularization Scheme ( $\chi R S$ for short). In this scheme, we can use chiral symmetry in order to establish some extra selection rules for the renormalization of the operators of interest. Although the resulting "operator renormalization" is incomplete on the lattice, it is the one which should be recovered in the the continuum limit, since the renormalized theory must have the desired chiral properties. The remaining lattice subtractions are due to the presence of the Wilson term.

In order to derive the selection rules in the $\chi R S$ scheme, it is adequate to consider two discrete axial symmetries. The first (denoted by $\chi_{24}$ ) acts only on flavours 2 and 4:

$$
\begin{array}{lll}
\psi_{2} \rightarrow i \gamma_{5} \psi_{2} & ; & \bar{\psi}_{2} \rightarrow i \bar{\psi}_{2} \gamma_{5} \\
\psi_{4} \rightarrow i \gamma_{5} \psi_{4} & ; & \bar{\psi}_{4} \rightarrow i \bar{\psi}_{4} \gamma_{5}
\end{array}
$$




\begin{tabular}{|r|c|c|c|c|c|c|c|c|c|c|}
\hline Symmetry & $Q_{1}^{+}$ & $Q_{2}^{+}$ & $Q_{3}^{+}$ & $Q_{4}^{+}$ & $Q_{5}^{+}$ & $Q_{1}^{-}$ & $Q_{2}^{-}$ & $Q_{3}^{-}$ & $Q_{4}^{-}$ & $Q_{5}^{-}$ \\
\hline \hline$\chi_{24}$ & -1 & +1 & +1 & -1 & -1 & -1 & +1 & +1 & -1 & -1 \\
\hline$\chi_{12}$ & +1 & $Q_{2}^{-}$ & $-Q_{3}^{-}$ & -1 & -1 & +1 & $Q_{2}^{+}$ & $-Q_{3}^{+}$ & -1 & -1 \\
\hline
\end{tabular}

Table 2: Classification of four-fermion parity conserving operators $Q_{k}^{ \pm}(k=1, \ldots, 5)$, according to the discrete symmetries $\chi_{24}$ and $\chi_{12}$. The parity violating operators $\mathcal{Q}_{k}^{ \pm}(k=1, \ldots, 5)$ transform in the same way.

The second symmetry (denoted as $\chi_{12}$ ) acts only on flavours 1 and 2:

$$
\begin{array}{lll}
\psi_{1} \rightarrow i \gamma_{5} \psi_{1} & ; & \bar{\psi}_{1} \rightarrow i \bar{\psi}_{1} \gamma_{5} \\
\psi_{2} \rightarrow i \gamma_{5} \psi_{2} & ; & \bar{\psi}_{2} \rightarrow i \bar{\psi}_{2} \gamma_{5}
\end{array}
$$

Under these transformations, the four-fermion operators transform as shown in Table 2 . The symmetry $\chi_{12}$ implies that $Q_{1}^{ \pm}$renormalize multiplicatively, whereas $Q_{4}^{+}$and $Q_{5}^{+}$ $\left(Q_{4}^{-}\right.$and $\left.Q_{5}^{-}\right)$mix with each other. From the $\chi_{24}$ symmetry we deduce that $Q_{2}^{+}$and $Q_{3}^{+}\left(Q_{2}^{-}\right.$and $\left.Q_{3}^{-}\right)$also mix with each other. In the absence of explicit chiral symmetry breaking, we conclude that the mixing structure is the same as that considered above for the parity-violating counterparts. The corresponding parity conserving operators $\left(Q_{k}^{ \pm} ; k=1, \ldots, 5\right)$, belonging to the same chiral representations, would have a $\chi R S$ mixing pattern similar to that of eq. (19):

$$
\left(\begin{array}{c}
\hat{Q}_{1} \\
\hat{Q}_{2} \\
\hat{Q}_{3} \\
\hat{Q}_{4} \\
\hat{Q}_{5}
\end{array}\right)^{ \pm}=\left(\begin{array}{rrrrr}
Z_{11} & 0 & 0 & 0 & 0 \\
0 & Z_{22} & Z_{23} & 0 & 0 \\
0 & Z_{32} & Z_{33} & 0 & 0 \\
0 & 0 & 0 & Z_{44} & Z_{45} \\
0 & 0 & 0 & Z_{54} & Z_{55}
\end{array}\right)^{ \pm}\left(\begin{array}{c}
\tilde{Q}_{1} \\
\tilde{Q}_{2} \\
\tilde{Q}_{3} \\
\tilde{Q}_{4} \\
\tilde{Q}_{5}
\end{array}\right)^{ \pm}
$$

where the $\tilde{Q}_{i}$ represent the bare operators in the $\chi R S$ scheme. In the presence of the Wilson term, the $\tilde{Q}_{i}$ are the lattice subtracted operators defined as

$$
\left(\begin{array}{l}
\tilde{Q}_{1} \\
\tilde{Q}_{2} \\
\tilde{Q}_{3} \\
\tilde{Q}_{4} \\
\tilde{Q}_{5}
\end{array}\right)^{ \pm}=\left(\begin{array}{l}
Q_{1} \\
Q_{2} \\
Q_{3} \\
Q_{4} \\
Q_{5}
\end{array}\right)^{ \pm}+\left(\begin{array}{rrrrr}
0 & \Delta_{12} & \Delta_{13} & \Delta_{14} & \Delta_{15} \\
\Delta_{21} & 0 & 0 & \Delta_{24} & \Delta_{25} \\
\Delta_{31} & 0 & 0 & \Delta_{34} & \Delta_{35} \\
\Delta_{41} & \Delta_{42} & \Delta_{43} & 0 & 0 \\
\Delta_{51} & \Delta_{52} & \Delta_{53} & 0 & 0
\end{array}\right)^{ \pm}\left(\begin{array}{l}
Q_{1} \\
Q_{2} \\
Q_{3} \\
Q_{4} \\
Q_{5}
\end{array}\right)^{ \pm}
$$

In other words, first the lattice subtraction is performed, followed by the $\chi R S$ renormalization. The above mixing pattern is abbreviated, in matrix form, as

$$
\begin{aligned}
\hat{Q}^{ \pm} & =Z_{\chi}^{ \pm} \tilde{Q}^{ \pm} \\
\tilde{Q}^{ \pm} & =\left[I+\Delta^{ \pm}\right] Q^{ \pm}
\end{aligned}
$$


where $I$ is the $5 \times 5$ unit matrix and the subscript $\chi$ stands for $\chi R S$ subtractions 7 . The renormalization of the parity conserving sector is given by $Z^{ \pm}=Z_{\chi}^{ \pm}\left[I+\Delta^{ \pm}\right]$. Note that, using continuous chiral transformations, in the hypothetical $\chi R S$, it is easy to show that $\Delta^{ \pm}=0$ and $\mathcal{Z}_{i j}=Z_{i j}$.

\section{NP renormalization: the lattice RI renormal- ization scheme}

In this section, we outline the strategy used in the determination of the renormalization constants, and discuss the appropriate renormalization conditions. According to the NP method proposed in [20], the renormalization conditions are imposed in momentum space on the projected amputated Green's functions. In this work we will always consider Green's functions where all external quark legs have the same momentum $p$. This choice is, of course, not unique but is the simplest way to regulate the infrared divergences. An important point is that the renormalization conditions need to be imposed at large Euclidean $p^{2}$. In the determination of the mixing coefficients this condition enables us to neglect soft or spontaneous chiral symmetry breaking effects which are not induced by the Wilson term and cannot be computed. As demonstrated in sec. 5, only at large external momenta is the NP method, described below, equivalent to the WI one. The large $p^{2}$ condition is also necessary in the evaluation of the overall renormalization constants, since the standard procedure of obtaining physical amplitudes from renormalized matrix elements requires perturbative matching with the Wilson coefficients in the continuum, at fixed gauge coupling. We will describe in subsect. 4.3 an alternative procedure, for which this condition can be relaxed.

The renormalization method presented in this section is an extension of the one introduced in [21] for the renormalization of the $O^{\Delta S=2}$ four-fermion operator. As in ref. [21], we will define projected-amputated Green functions, on which suitable renormalization conditions will be imposed. With respect to ref. [21], the novelties consist in using a complete operator basis for the parity-conserving sector and in extending the method to the parity-violating operators.

\subsection{Amputated Green functions and their projectors}

We first give some general definitions. Since the non-perturbative renormalization conditions are to be imposed on quark states, we shall need the general expression of the four-point Green's functions of the operators $O_{\Gamma^{(1)} \Gamma^{(2)}}^{ \pm}$. Denoting by $x_{1}, x_{3}$ and $x_{2}, x_{4}$ the coordinates of the outgoing and incoming quarks respectively, we define the

\footnotetext{
${ }^{7}$ Attention is drawn to the notation adopted from now on in this paper: whereas the elements of matrices $\mathcal{Z}^{ \pm}$and $\Delta^{ \pm}$are denoted, in standard fashion, as $\mathcal{Z}_{i j}^{ \pm}$and $\Delta_{i j}^{ \pm}$, those of matrix $Z_{\chi}^{ \pm}$are denoted by $Z_{i j}^{ \pm}$(i.e. the subscript $\chi$ is dropped for notational economy). The elements of matrix $Z^{ \pm}$, appearing in eq. (17), will never be used from now on.
} 
connected one-particle-irreducible Green's functions as

$$
G_{\Gamma^{(1)} \Gamma^{(2)}}^{ \pm}\left(x_{1}, x_{2}, x_{3}, x_{4}\right)=\left\langle\psi_{1}\left(x_{1}\right) \bar{\psi}_{2}\left(x_{2}\right) O_{\Gamma^{(1)} \Gamma^{(2)}}^{ \pm}(0) \psi_{3}\left(x_{3}\right) \bar{\psi}_{4}\left(x_{4}\right)\right\rangle,
$$

where $\langle\cdots\rangle$ denotes the vacuum expectation value, i.e. the average over the gauge-field configurations. The generic four-fermion operator $O_{\Gamma^{(1)} \Gamma^{(2)}}$, placed at the origin, is written as

$$
\begin{aligned}
& O_{\Gamma^{(1)} \Gamma^{(2)}}^{ \pm}(0)= \\
& \frac{1}{2}\left[\bar{\psi}_{1}(0) \Gamma^{(1) m} \psi_{2}(0) \bar{\psi}_{3}(0) \Gamma^{(2) m} \psi_{4}(0) \pm \bar{\psi}_{1}(0) \Gamma^{(1) m} \psi_{4}(0) \bar{\psi}_{3}(0) \Gamma^{(2) m} \psi_{2}(0)\right] .
\end{aligned}
$$

Given the complexity of the notation, we shall elaborate on this formula. In the case of parity conserving operators, the two Dirac matrices are equal; i.e. we are dealing with the $O_{\Gamma \Gamma}^{ \pm}$of eq. (11). The case of two different Dirac matrices $\Gamma^{(1)} \neq \Gamma^{(2)}$ applies to the parity violating operators of eqs. (13),(14) and (15), for which, for example, $\Gamma^{(1)}=V$ and $\Gamma^{(2)}=A$. Note that $G_{\Gamma^{(1)} \Gamma^{(2)}}^{ \pm}$depends implicitly on the four colour and Dirac indices carried by the external fermion fields. These will be shown explicitly from now on. Following the conventions of Appendix A, upper-case Latin superscripts $(A, B, \ldots, R, S, \ldots)$ will denote colour indices of the fundamental $\mathrm{SU}(3)$ representation. Greek lower-case subscripts $(\alpha, \beta, \ldots, \rho, \sigma, \ldots)$ will denote spinor indices. The letters $m$ and $n$ are reserved for the Lorentz indices of the Dirac matrices. Repeated Lorentz indices are summed according to the convention adopted in Appendix A.

The Fourier transform of the non-amputated Green's function (25), at equal external momenta $p$, has the form

$$
G_{\Gamma^{(1)} \Gamma^{(2)}}^{ \pm}(p)_{\alpha \beta \gamma \delta}^{A B C D}=\frac{1}{2}\left[\left\langle\Gamma^{(1) m}(p)_{\alpha \beta}^{A B} \Gamma^{(2) m}(p)_{\gamma \delta}^{C D}\right\rangle \mp\left\langle\Gamma^{(1) m}(p)_{\alpha \delta}^{A D} \Gamma^{(2) m}(p)_{\gamma \beta}^{C B}\right\rangle\right],
$$

where

$$
\Gamma^{(i) m}(p)_{\alpha \beta}^{A B}=S(p \mid 0)_{\alpha \rho}^{A R} \Gamma_{\rho \sigma}^{(i) m}\left[\gamma_{5} S(p \mid 0)^{\dagger} \gamma_{5}\right]_{\sigma \beta}^{R B}, \quad(i=1,2)
$$

and $S(p \mid 0)$ is defined by

$$
S(p \mid 0)=\int d^{4} x S(x \mid 0) e^{-i p \cdot x} .
$$

$S(x \mid 0)$ is the inverse of the lattice Dirac operator; i.e. it is the "quark propagator" computed on a single gauge-field configuration and is therefore not translationally invariant (cf. section 4 of ref. 20]). It satisfies the relation

$$
S(x \mid 0)=\gamma_{5} S^{\dagger}(0 \mid x) \gamma_{5} .
$$

The amputated Green's function is obtained from eq. (27)

$$
\Lambda_{\Gamma^{(1)} \Gamma^{(2)}}^{ \pm}(p)_{\rho \sigma \rho^{\prime} \sigma^{\prime}}^{R S R^{\prime} S^{\prime}}=S^{-1}(p)_{\rho \alpha}^{R A} S^{-1}(p)_{\rho^{\prime} \gamma}^{R^{\prime} C} G_{\Gamma^{(1)} \Gamma^{(2)}}^{ \pm}(p)_{\alpha \beta \gamma \delta}^{A B C D} S^{-1}(p)_{\beta \sigma}^{B S} S^{-1}(p)_{\delta \sigma^{\prime}}^{D S^{\prime}}
$$

where $S(p)=\langle S(p \mid 0)\rangle$ is the Fourier transform of the translationally invariant quark propagator, i.e. the Fourier transform of $S(x \mid 0)$, averaged over the gauge-field configurations. 
The amputated Green's function of eq. (31) is a high-rank tensor, from which a more manageable function of the external momenta $p$ can be obtained by projecting over all the possible Dirac structures. Let us introduce a generic Dirac projector

$$
\mathrm{P}_{\hat{\Gamma}^{(1)} \hat{\Gamma}^{(2)}} \equiv\left(\hat{\Gamma}^{(1) n} \otimes \hat{\Gamma}^{(2) n}\right),
$$

Its application on the amputated Green's functions is defined as ${ }^{8}$ :

$$
\operatorname{Tr} \mathbf{P}_{\hat{\Gamma}^{(1)} \hat{\Gamma}^{(2)}} \Lambda_{\Gamma^{(1)} \Gamma^{(2)}}^{ \pm}(p)=\left(\hat{\Gamma}_{\sigma \rho}^{(1) n} \otimes \hat{\Gamma}_{\sigma^{\prime} \rho^{\prime}}^{(2) n}\right) \Lambda_{\Gamma^{(1)} \Gamma^{(2)}}^{ \pm}(p)_{\rho \sigma \rho^{\prime} \sigma^{\prime}}^{R R R^{\prime} R^{\prime}}
$$

The trace is taken over spin and colour. The projectors $\mathrm{P}_{\hat{\Gamma}^{(1)} \hat{\Gamma}^{(2)}}$ can be worked out analytically for the tree-level amputated Green's function

$$
\Lambda_{\Gamma^{(1)} \Gamma^{(2)}}^{ \pm(0)}(p)_{\rho \sigma \rho^{\prime} \sigma^{\prime}}^{R S R^{\prime} S^{\prime}}=\frac{1}{2}\left[\delta^{R S} \delta^{R^{\prime} S^{\prime}}\left(\Gamma_{\rho \sigma}^{(1) m} \otimes \Gamma_{\rho^{\prime} \sigma^{\prime}}^{(2) m}\right) \mp \delta^{R S^{\prime}} \delta^{R^{\prime} S}\left(\Gamma_{\rho \sigma^{\prime}}^{(1) m} \otimes \Gamma_{\rho^{\prime} \sigma}^{(2) m}\right)\right]
$$

The superscript (0) denotes tree-level. From eqs. (33) and (34) one finds

$$
\begin{aligned}
\operatorname{Tr} \mathrm{P}_{\hat{\Gamma}^{(1)} \hat{\Gamma}^{(2)}} \Lambda_{\Gamma^{(1)} \Gamma^{(2)}}^{ \pm(0)}(p)= & \left(\hat{\Gamma}_{\sigma \rho}^{(1) n} \otimes \hat{\Gamma}_{\sigma^{\prime} \rho^{\prime}}^{(2) n} \Lambda_{\Gamma^{(1)} \Gamma^{(2)}}^{ \pm(0)}(p)_{\rho \sigma \rho^{\prime} \sigma^{\prime}}^{R R R^{\prime}}\right. \\
= & \frac{1}{2}\left[N_{c}^{2}\left(\operatorname{Tr} \hat{\Gamma}^{(1) n} \Gamma^{(1) m}\right)\left(\operatorname{Tr} \hat{\Gamma}^{(2) n} \Gamma^{(2) m}\right)\right. \\
& \left.\mp N_{c}\left(\operatorname{Tr} \hat{\Gamma}^{(1) n} \Gamma^{(1) m} \hat{\Gamma}^{(2) n} \Gamma^{(2) m}\right)\right]
\end{aligned}
$$

These formulae can now be used in the specific cases of interest, namely the renormalization of the various parity violating and parity conserving operators (i.e. $Q_{k}^{ \pm}$ of eq. (12) and $\mathcal{Q}_{k}^{ \pm}$of eq. (16) with $\left.k=1, \ldots, 5\right)$. We will see shortly that, for the renormalization conditions we will impose on the quark correlation functions of the four-fermion operators, it is convenient to use projectors that obey the following orthogonality conditions:

$$
\begin{aligned}
\operatorname{Tr} \mathrm{P}_{i}^{ \pm} \Lambda_{k}^{ \pm(0)} & =\delta_{i k} & & (i, k=1, \ldots, 5) \\
\operatorname{Tr} \mathrm{P}_{i}^{ \pm} \mathcal{J}_{k}^{ \pm(0)} & =\delta_{i k} & & (i, k=1, \ldots, 5)
\end{aligned}
$$

where $\Lambda_{k}^{(0) \pm}$ and $\mathcal{X}_{k}^{(0) \pm}$ are the tree-level amputated Green functions of operators $Q_{k}^{ \pm}$and $\mathcal{Q}_{k}^{ \pm}$respectively $(k=1, \ldots, 5)$, and $\mathrm{P}_{k}^{ \pm}, \mathrm{p}_{k}^{ \pm}$their corresponding projectors $(k=1, \ldots, 5)$. In the following we will denote as $\Lambda_{k}^{ \pm}$and $\mathcal{X}_{k}^{ \pm}$the corresponding Green functions in the interacting case. With the aid of eq. (35) we find for the parity conserving case

$$
\begin{aligned}
& \mathbf{P}_{1}^{ \pm} \equiv+\frac{1}{64 N_{c}\left(N_{c} \pm 1\right)}\left(\mathbf{P}_{V V}+\mathbf{P}_{A A}\right) \\
& \mathbf{P}_{2}^{ \pm} \equiv+\frac{1}{64\left(N_{c}^{2}-1\right)}\left(\mathbf{P}_{V V}-\mathbf{P}_{A A}\right) \pm \frac{1}{32 N_{c}\left(N_{c}^{2}-1\right)}\left(\mathbf{P}_{S S}-\mathbf{P}_{P P}\right)
\end{aligned}
$$

\footnotetext{
${ }^{8}$ Other possible choices are admissible; see [2].
} 


$$
\begin{aligned}
& \mathbf{P}_{3}^{ \pm} \equiv \pm \frac{1}{32 N_{c}\left(N_{c}^{2}-1\right)}\left(\mathbf{P}_{V V}-\mathbf{P}_{A A}\right)+\frac{1}{16\left(N_{c}^{2}-1\right)}\left(\mathbf{P}_{S S}-\mathbf{P}_{P P}\right) \\
& \mathbf{P}_{4}^{ \pm} \equiv+\frac{\left(2 N_{c} \pm 1\right)}{32 N_{c}\left(N_{c}^{2}-1\right)}\left(\mathbf{P}_{S S}+\mathbf{P}_{P P}\right) \mp \frac{1}{32 N_{c}\left(N_{c}^{2}-1\right)} \mathbf{P}_{T T} \\
& \mathbf{P}_{5}^{ \pm} \equiv \mp \frac{1}{32 N_{c}\left(N_{c}^{2}-1\right)}\left(\mathbf{P}_{S S}+\mathbf{P}_{P P}\right)+\frac{\left(2 N_{c} \mp 1\right)}{96 N_{c}\left(N_{c}^{2}-1\right)} \mathbf{P}_{T T}
\end{aligned}
$$

whereas for the parity violating one

$$
\begin{aligned}
& \wp_{1}^{ \pm} \equiv-\frac{1}{64 N_{c}\left(N_{c} \pm 1\right)}\left(\mathbf{P}_{V A}+\mathbf{P}_{A V}\right) \\
& \Upsilon_{2}^{ \pm} \equiv-\frac{1}{64\left(N_{c}^{2}-1\right)}\left(\mathbf{P}_{V A}-\mathbf{P}_{A V}\right) \mp \frac{1}{32 N_{c}\left(N_{c}^{2}-1\right)}\left(\mathbf{P}_{S P}-\mathbf{P}_{P S}\right) \\
& \Upsilon_{3}^{ \pm} \equiv \mp \frac{1}{32 N_{c}\left(N_{c}^{2}-1\right)}\left(\mathbf{P}_{V A}-\mathbf{P}_{A V}\right)-\frac{1}{16\left(N_{c}^{2}-1\right)}\left(\mathbf{P}_{S P}-\mathbf{P}_{P S}\right) \\
& \wp_{4}^{ \pm} \equiv+\frac{\left(2 N_{c} \pm 1\right)}{32 N_{c}\left(N_{c}^{2}-1\right)}\left(\mathbf{P}_{S P}+\mathbf{P}_{P S}\right) \mp \frac{1}{32 N_{c}\left(N_{c}^{2}-1\right)} \mathbf{P}_{T \tilde{T}} \\
& \wp_{5}^{ \pm} \equiv \mp \frac{1}{32 N_{c}\left(N_{c}^{2}-1\right)}\left(\mathbf{P}_{S P}+\mathbf{P}_{P S}\right)+\frac{\left(2 N_{c} \mp 1\right)}{96 N_{c}\left(N_{c}^{2}-1\right)} \mathbf{P}_{T \tilde{T}}
\end{aligned}
$$

\subsection{Renormalization conditions}

We now define the renormalization procedure of the operators of interest. The general principle is to impose "suitable" renormalization conditions, which are satisfied by the renormalized (projected amputated) Green functions $\hat{\Lambda}^{ \pm}$and $\hat{\mathcal{X}}^{ \pm}$at a fixed scale $\mu$ in the deep Euclidean region. The renormalization condition is arbitrary. A simple choice

is to impose that the fully interacting $\hat{\Lambda}^{ \pm}$(and $\hat{\mathcal{\lambda}}^{ \pm}$), at a given scale $\mu$, are equal to their tree level values written in eq. (35); see also eqs. (36).

We will use matrix notation for simplicity: the amputated-projected Green functions $\Lambda^{ \pm}$and $\mathcal{J}^{ \pm}$denote $1 \times 5$ row vectors, whereas the projectors $\mathrm{P}^{ \pm}$and $\mathrm{p}^{ \pm}$denote $5 \times 1$ column vectors. In this notation, eqs. $(36)$ become

$$
\begin{aligned}
\mathrm{P}^{ \pm} \cdot \Lambda^{ \pm(0)} & =I \\
\mathrm{P}^{ \pm} \cdot \mathcal{\lambda}^{ \pm(0)} & =I
\end{aligned}
$$

with $I$ the $5 \times 5$ unit matrix. For the parity-violating case, the renormalized amputated Green's function is given by the row vector

$$
\hat{\mathcal{X}}^{ \pm}\left(p / \mu, g^{2}\right)=Z_{\psi}^{-2}\left(a \mu, g_{0}^{2}\right) \mathcal{J}^{ \pm}\left(a p, g_{0}^{2}\right) \mathcal{Z}^{ \pm}\left(a \mu, g_{0}^{2}\right)^{T}
$$

where $Z_{\psi}$ is the quark field renormalization constant and $\mathcal{Z}^{ \pm}$is the $5 \times 5$ renormalization matrix (the superscript $T$ stands for transpose). Recall that $\mathcal{Z}^{ \pm}$is the block diagonal matrix of eq. (19). In the above expression we denote by $g_{0}^{2} \equiv g_{0}^{2}(a)$ the bare coupling 
and by $g^{2} \equiv g^{2}(\mu)$ the renormalized one. We express the bare Green function in terms of a "dynamics" matrix $\mathcal{D}$ from which the tree-level amputated Green's function is factored out:

$$
\mathcal{N}^{ \pm}=\mathcal{X}^{ \pm(0)} \mathcal{D}^{ \pm}
$$

Note that since the matrix $\mathcal{D}$ determines the "dynamics" of the bare operators, it can only mix tree-level operators with the same discrete symmetries. Thus, it is also a block diagonal matrix with the same block structure as $\mathcal{Z}^{ \pm}$. From eqs. (39) and (41) the elements of the matrix $\mathcal{D}^{ \pm}$are expressed in terms of the amplitudes $\mathcal{X}^{ \pm}$:

$$
\mathcal{D}^{ \pm}=\wp^{ \pm} \cdot \mathcal{J}^{ \pm}
$$

We compute $\mathcal{J}^{ \pm}$non-perturbatively, at fixed coupling $g_{0}^{2}$ and in a given gauge, over a configuration ensemble. We opt for the Landau gauge. From $\mathcal{J}^{ \pm}$and eq. (42), we obtain $\mathcal{D}^{ \pm}$.

The renormalization conditions

$$
\left.p^{ \pm} \cdot \hat{\mathcal{X}}^{ \pm}\left(p / \mu, g^{2}\right)\right|_{p^{2}=\mu^{2}}=I
$$

determine the mixing matrix $\mathcal{Z}^{ \pm}$. This is easily seen by combining eqs. (39), (40), (42) and (43) to obtain

$$
Z_{\psi}^{ \pm-2} \mathcal{D}^{ \pm} \mathcal{Z}^{ \pm T}=I
$$

from which we obtain $\mathcal{Z}^{ \pm}$in terms of the known quantities $Z_{\psi}^{ \pm 2}$ and $\mathcal{D}^{ \pm}$:

$$
\mathcal{Z}^{ \pm}=Z_{\psi}^{ \pm 2}\left[\mathcal{D}^{ \pm T}\right]^{-1}
$$

Note that since both $\mathcal{D}^{ \pm}$and $\mathcal{Z}^{ \pm}$have the same block diagonal structure, this involves inverting at most $2 \times 2$ matrices.

The quark field renormalization $Z_{\psi}$ is also determined non-perturbatively from the numerical simulation. A definition which respects WI's (cf. sec. 4 of ref. [20] for details) is

$$
Z_{\psi}=-\left.i \frac{1}{12} \operatorname{Tr} \gamma_{\mu} \frac{\partial S(p)^{-1}}{\partial p_{\mu}}\right|_{p^{2}=\mu^{2}}
$$

Instead, we have implemented

$$
Z_{\psi}(\mu a)=-\left.i \frac{1}{12} \frac{\sum_{\mu=1,4} \gamma_{\mu} \sin \left(p_{\mu} a\right) S(p a)^{-1}}{4 \sum_{\mu=1,4} \gamma_{\mu} \sin ^{2}\left(p_{\mu} a\right)}\right|_{p^{2}=\mu^{2}}
$$

in order to avoid derivatives w.r.t. discrete variables.

The computation of the parity-conserving matrix $Z^{ \pm}$follows similar lines. Recall that this matrix can be cast in the form $Z_{\chi}^{ \pm}\left[I+\Delta^{ \pm}\right]$, with $Z_{\chi}^{ \pm}$the block diagonal matrix of eq. (22) and $\Delta^{ \pm}$the sparse matrix of eq. (23). We find it convenient to 
obtain $Z_{\chi}^{ \pm}$and $\Delta^{ \pm}$separately. As we will show in the next section, $Z_{\chi}^{ \pm}$depends on the renormalization scale $a \mu$, whereas $\Delta^{ \pm}$does not. More importantly, as we will also show in sect. 5, the independence of $\Delta^{ \pm}$from $a \mu$ is due to the fact that the lattice subtractions can also be determined from WI's, in the spirit of ref. [18]. Thus, our results on $\Delta^{ \pm}$, obtained in the RI scheme with the NP method, can in principle be compared to those obtained with WI's.

For the parity-conserving case, the fully interacting renormalized amputated Green's function is given by the row $1 \times 5$ vector

$$
\hat{\Lambda}^{ \pm}\left(p / \mu, g^{2}\right)=Z_{\psi}^{-2}\left(a \mu, g_{0}^{2}\right) \Lambda^{ \pm}\left(a p, g_{0}^{2}\right)\left[I+\Delta^{ \pm}\left(g_{0}^{2}\right)^{T}\right] Z_{\chi}^{ \pm}\left(a \mu, g_{0}^{2}\right)^{T}
$$

We express the bare Green's function in terms of a "dynamics" matrix $D^{ \pm}$:

$$
\Lambda^{ \pm}=\Lambda^{ \pm(0)} D^{ \pm}
$$

From eqs. (39) and (49) the elements of the matrix $D^{ \pm}$are expressed in terms of the amplitudes $\Lambda^{ \pm}$:

$$
D^{ \pm}=\mathrm{P}^{ \pm} \cdot \Lambda^{ \pm}
$$

Therefore $D^{ \pm}$can be computed non-perturbatively by numerical simulation, at fixed bare coupling $g_{0}^{2}$, over a configuration ensemble and in the Landau gauge. Once it is computed, we determine the mixing matrix $\Delta^{ \pm}$and the renormalization matrix $Z_{\chi}^{ \pm}$ from the renormalization conditions

$$
\left.\mathbf{P}^{ \pm} \cdot \hat{\Lambda}^{ \pm}\left(p / \mu, g^{2}\right)\right|_{p^{2}=\mu^{2}}=I
$$

To see this explicitly, we combine eqs.(39), (48), (49) and (50), in order to express eq.(51) as follows:

$$
Z_{\psi}^{-2} D^{ \pm}\left[I+\Delta^{ \pm T}\right] Z_{\chi}^{ \pm T}=I
$$

We then proceed in two steps. Let us first rewrite the above expression as

$$
D_{k l}^{ \pm}+\sum_{j=1}^{5} D_{k j}^{ \pm} \Delta_{l j}^{ \pm}=Z_{\psi}^{2}\left[Z_{\chi}^{ \pm-1}\right]_{l k}
$$

(in matrix-component notation). We then consider the special case, with indices $k, l$ chosen so that for a given fixed value $k$, the index $l$ is allowed to run over the corresponding "lattice subtracted" values. For example, if we fix $k=1, l$ runs over the range $l=2,3,4,5$; if we fix $k=2$ or $k=3, l$ runs over the range $l=1,4,5$ etc. With these choices of $k, l$, and given the structure of matrix $\Delta^{ \pm}$(c.f. eq.(23)), the summed index $j$ also runs over the same interval as $l$ (all other contributions involve zero matrix elements of $\Delta^{ \pm}$). Moreover, the r.h.s. vanishes for these combinations of $k, l$, due to the block-diagonal structure of the matrix $Z_{\chi}^{ \pm}$(c.f. eq.(22)). Defining the column vector $c_{l}^{ \pm}=D_{k l}^{ \pm}$for fixed $k$ and $l$ running as detailed above, we obtain the equation

$$
\Delta_{l j}^{ \pm} c_{j}^{ \pm}=-c_{l}^{ \pm}
$$


This is a linear inhomogenous system, which can be solved for $\Delta_{l j}^{ \pm}$. Thus, the mixing matrix is determined, and the subtracted correlation function can be constructed. We can now proceed to the second step, which is the determination of the renormalization matrix $Z_{\chi}^{ \pm}$. It can be obtained exactly like in the parity violating case; cf. eqs. (44) and (45).

\subsection{Range of validity of the RI renormalization scheme}

Having completed our discussion on the determination of the renormalization constants and mixing coefficients of four-fermion operators, using the NP method of ref. [20], we now summarize the renormalization conditions. For the parity violating operators we have

$$
\left.Z_{\psi}^{-2} \operatorname{Tr} p^{ \pm} \cdot \mathcal{N}^{ \pm} \mathcal{Z}^{ \pm T}\right|_{p^{2}=\mu^{2}}=I
$$

For the parity conserving operators we have

$$
\begin{aligned}
& \left.Z_{\psi}^{-2} \operatorname{Tr} \mathrm{P}^{ \pm} \cdot \Lambda^{ \pm} Z^{ \pm T}\right|_{p^{2}=\mu^{2}}=\left.Z_{\psi}^{-2} \operatorname{Tr} \mathbf{P}^{ \pm} \cdot \tilde{\Lambda}^{ \pm} Z_{\chi}^{ \pm T}\right|_{p^{2}=\mu^{2}}= \\
& \left.Z_{\psi}^{-2} \operatorname{Tr} \mathbf{P}^{ \pm} \cdot \Lambda^{ \pm}\left[I+\Delta^{ \pm T}\right] Z_{\chi}^{ \pm T}\right|_{p^{2}=\mu^{2}}=I
\end{aligned}
$$

with $Z_{\psi}$ determined from eq. (47). In the last equation, $\tilde{\Lambda}^{ \pm}$denotes the amputated Green's function for the lattice-subtracted parity conserving operators. These conditions constitute a renormalization scheme, called the Regularization Independent (RI)

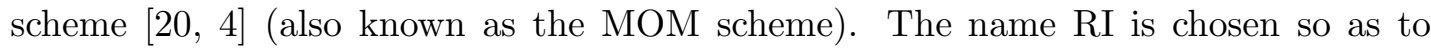
distinguish it from the $\overline{\mathrm{MS}}$ scheme, adopted in perturbation theory, which depends on the detailed choice of dimensional regularization ('t Hooft-Veltman, Dimensional Reduction, etc.). In the RI scheme, the renormalized operator depends on the gauge and on the momenta of the external states [20, 21]. Unlike the $\overline{\mathrm{MS}}$, the RI scheme does not depend on the regularization. Since the renormalized matrix element must be multiplied by the Wilson coefficient $C_{W}(\mu)$ (cf. eq. (1)) the latter must also be calculated in the same gauge and with the same external states in order to obtain a renormalization group invariant result. The Wilson coefficient is known in next-to-leading order continuum PT 四; thus, its matching with the renormalized matrix element is accurate to that order. Clearly, for the perturbative calculation of $C_{W}(\mu)$ to be reliable, we must ensure that $\mu \gg \Lambda_{\mathrm{QCD}}$.

There is also another reason for which this condition must be imposed: the RI conditions are such that the renormalized operators transform according to irreducible representations of the chiral algebra. This is achieved by imposing that the projections of the renormalized operators on the "wrong" chiral structures vanish (cf. eq.(56)). But this not true when there are other causes of chiral symmetry breaking, either due to the 
explicit presence of mass terms or due to spontaneous symmetry breaking in the chiral limit. Such effects, present in QCD, would modify the r.h.s. of the renormalization conditions (55) and (56) by form factors proportional to the quark mass $m$, the chiral condensate $\langle\bar{\psi} \psi\rangle$ and the inverse scale $\mu^{-1}$. These terms die off in the large momenta region, $\mu \gg \Lambda_{\mathrm{QCD}}$.

On the other hand, the non-perturbative renormalization of the four-fermion operator in the RI scheme involves computations of bare matrix elements at finite lattice cut-off. In order to have good control of the discretization errors, we must also ensure that $\mu \ll \mathcal{O}\left(a^{-1}\right)$. Thus, as already pointed out in [20], the RI scheme is applicable at couplings $g_{0}^{2}$ and lattice sizes for which there exists a window of $\mu$ values satisfying

$$
\Lambda_{\mathrm{QCD}} \ll \mu \ll \mathcal{O}\left(a^{-1}\right)
$$

The discussion leading to the necessity of the above window of $\mu$ values is based on the assumption that the operator renormalization is performed at fixed UV cutoff (i.e. the gauge coupling is fixed in the numerical computation). One can relax the bound of eq. (57) by performing a sequence of computations at bare couplings $g_{0}(a), g_{0}(s a)$, $g_{0}\left(s^{2} a\right), \cdots$ corresponding to increasingly coarse lattice spacings (i.e. the scaling factor $s>1$; typically $s=2$ ). This method of lowering the renormalization scale $\mu$ in a controlled NP way is reminiscent of the renormalization procedure of ref. [26], which in turn was inspired by refs. [19]. We outline it here in schematic fashion; wave function renormalization, operator subtractions etc. will be omitted for the sake of clarity. Realistically, with present day resources, this procedure can be iterated only two or three times.

We first select a small coupling constant $g_{0}^{2}$, so that we are safely in the perturbative region (i.e. $g_{0}^{2}$ is smaller than current values in standard QCD simulations). For such small lattice spacings, the window of eq. (57) is easily satisfied by both momentum scales $\mu$ and $\mu / s$ used in the following. We now chose a lattice of $N$ lattice sites in each direction ( $N$ is only limited by current computational capabilities), in such a way that $a \mu \ll 1$, in order to avoid (UV) lattice artifacts, and at the same time large enough to have negligible finite size effects; i.e. $a \mu / s \gg 1 / N$. With small $g_{0}^{2} \sim 0.7$, and $N \sim 32$ 48 , this lattice is adequate for simulations of quark correlation functions deep in the perturbative region, but cannot accomodate hadronic quantities, due to the smallness of its physical volume. Once the RI scheme has been implemented at the scale $\mu$, the renormalized correlation function of a given operator is known at any scale $p$ satisfying eq. (57):

$$
\hat{\Lambda}\left(\frac{p}{\mu}, g^{2}(\mu)\right)=Z\left(a \mu, g_{0}^{2}(a)\right) \Lambda\left(a p, g_{0}^{2}(a)\right)
$$

In particular, we define

$$
\hat{\Lambda}_{1} \equiv \hat{\Lambda}\left(\frac{1}{s}, g^{2}(\mu)\right)
$$

i.e. the renormalized vertex at a scale $p=\mu / s$. We now increase the lattice spacing to $a^{\prime}=s a$. This is done in practice by tuning the bare coupling $g_{0}^{2}$, on a bigger (coarser) 
lattice. For simplicity, since we assumed that finite size effects are negligible both at $\mu$ and $\mu / s$, we keep $N$ fixed. On the coarser lattice we compute the bare correlation function at momentum $p^{\prime}=\mu / s$ (i.e. for $a^{\prime} p^{\prime}=a \mu$ ). The renormalization constant at the scale $\mu$ (for cutoff $a^{\prime}$ ) can then be obtained from the equation

$$
\hat{\Lambda}_{1}=Z\left(a^{\prime} \mu, g_{0}^{2}\left(a^{\prime}\right)\right) \Lambda\left(a^{\prime} p^{\prime}, g_{0}^{2}\left(a^{\prime}\right)\right)
$$

since the l.h.s. of this expression is known from the calculation on the finer lattice (eq. (59)). With the renormalization constant $Z\left(a^{\prime} \mu, g_{0}^{2}\left(a^{\prime}\right)\right)$ thus obtained on the coarse lattice, we now compute the bare correlation function at momentum $p^{\prime \prime}=\mu / s^{2}$ (i.e. $\left.a^{\prime} p^{\prime \prime}=a \mu / s\right)$. For this momentum we then have

$$
\hat{\Lambda}\left(\frac{1}{s^{2}}, g^{2}(\mu)\right)=Z\left(a^{\prime} \mu, g_{0}^{2}\left(a^{\prime}\right)\right) \Lambda\left(a^{\prime} p^{\prime \prime}, g_{0}^{2}\left(a^{\prime}\right)\right)
$$

Thus the renormalized correlation function is now known at a lower momentum scale $1 / s^{2}$ :

$$
\Lambda_{2} \equiv \hat{\Lambda}\left(\frac{1}{s^{2}}, g^{2}(\mu)\right)
$$

One can now repeat the cycle: going to a coarser lattice $a^{\prime \prime}=s^{2} a$, we compute the bare correlation function at momentum $p^{\prime \prime}=\mu / s^{2}$ (i.e. $a^{\prime \prime} p^{\prime \prime}=a \mu$ ). The renormalization constant at scale $\mu$ is then obtained by solving

$$
\Lambda_{2}=Z\left(a^{\prime \prime} \mu, g_{0}^{2}\left(a^{\prime \prime}\right)\right) \Lambda\left(a^{\prime \prime} p^{\prime \prime}, g_{0}^{2}\left(a^{\prime \prime}\right)\right)
$$

for $Z\left(a^{\prime \prime} \mu, g_{0}^{2}\left(a^{\prime \prime}\right)\right)$. The bare correlation function is then computed at momentum $p^{\prime \prime \prime}=\mu / s^{3}$ (i.e. $a^{\prime \prime} p^{\prime \prime \prime}=a \mu / s$ ) and renormalized by $Z$ at scale $\mu$, giving the renormalized correlation function at scale $1 / s^{3}$ :

$$
\hat{\Lambda}\left(\frac{1}{s^{3}}, g^{2}(\mu)\right)=Z\left(a^{\prime \prime} \mu, g_{0}^{2}\left(a^{\prime \prime}\right)\right) \Lambda\left(a^{\prime \prime} p^{\prime \prime \prime}, g_{0}^{2}\left(a^{\prime \prime}\right)\right)
$$

At the end of the day, by keeping $N$ fixed, we end up with a lattice coarse enough to contain the hadrons, and an operator, renormalized non-perturbatively at a low scale $\mu / s^{n}$.

\section{$5 \quad$ Mixing coefficients from WI's}

In [18 an alternative approach to the NP renormalization method has been applied. This is based on lattice WI's on quark states, in the spirit of [11] and [12, 14]. The WI renormalization has been implemented in [18] for the non-perturbative evaluation of the mixing renormalization constants of the $\Delta S=2$ matrix element of $K^{0}-\bar{K}^{0}$ oscillations. In our language, this is the renormalization of the parity conserving operator $Q_{1}^{+}$. The WI's were derived in momentum space, the various chiral structures were projected 
out (just like in [21] and the present work) and they were solved for the mixing lattice coefficients $\Delta_{1 k}^{+}($for $k=2, \ldots, 5)$. The multiplicative renormalization constant $Z_{11}^{+}$was obtained using the NP method of [20, 21]. In principle, both methods are equivalent (under certain conditions, which will be discussed below) and suffer from the same sources of systematic error. In practice, one may give more stable results than the other. A systematic comparison of the two methods is an interesting problem; for the $\Delta S=2$ case it has been carried out in ref. [18].

Here we wish to extend this method to the complete basis of operators. We will therefore write down the WI's which can be used for the extraction of all mixing coefficients (i.e. all the elements of the matrix $\Delta^{ \pm}$of eq. (23)). We will also demonstrate explicitly that the WI method for the determination of the lattice mixing coefficients is equivalent to the RI method. This equivalence is true, up to discretization errors, provided the renormalization scale $\mu$ of the RI scheme is large (cf. eq. (57)) and that we are working in the chiral limit or with a mass independent scheme. The reasons for requiring a large $\mu$ for the RI scheme have been explained in subsect. 4.3. We now present briefly the reasons for which the implementation of the small $m$ limit for the WI scheme is required.

The WI holds for operators with the correct chiral properties, i.e. multiplicatively renormalizable operators transforming according to a well defined representation of the chiral algebra. By imposing its validity on the renormalized operators one can fix the mixing coefficients of the operators which stem from the chiral violation due to the Wilson term. This, however, is only true in the chiral limit, where chiral symmetry breaking is only due to the presence of the Wilson term in the lattice action. Upon introducing a mass term (soft symmetry breaking), the continuum WI's can only be recovered by the simultaneous redefinition of the $T$-product and the operator. This procedure is ambiguous, since a change in the definition of the $T$-product can be compensated by a redefinition of the operator. Thus, away from the chiral limit, the WI's do not uniquely define the operator (and, consequently, its mixing coefficients). This ambiguity is harmless if we can apply low-energy theorems of Current Algebra (small quark mass). This point has been discussed in ref. [11], and more recently in 42].

We will explicitly demonstrate in this section that the WI method and the RI renormalization scheme are equivalent methods for the determination of the lattice mixing coefficients, under the conditions discussed above. Moreover, we will show that the $\chi R S$ renormalization matrices $\mathcal{Z}$ and $Z_{\chi}$ cannot be determined from WI's. Only finite ratios of $\chi R S$ renormalization constants of opposite parity are fixed by the WI's. Thus the RI scheme (or some other renormalization method) is necessary for their determination, even when the WI method is used in order to obtain the lattice mixing coefficients. 


\subsection{Ward Identities on quark states}

The validity of the above statements has been shown, in a general and elegant way, in ref. [42]. Our aim is much more specific: we will obtain useful WI's which can be used in practice for the determination of the mixing coefficients of all four-fermion operators of interest. The above general statements will consequently be proved in the context of these specific WI's, which can be implemented in numerical simulation. In what follows, for reasons of uniformity of presentation, we will continue to work with operators which have four distinct flavours (unlike ref. [18], where the flavour group used was $S U(2)$ and the $\Delta S=2$ operator considered carried the strange and down physical flavours). The most economical way of obtaining useful WIs for the four flavour operators defined in eqs. (12) and (16) consists in the following trick. We consider an "embedding" of our operators (with four distinct flavours) in a theory with five flavours. In other words, our flavour symmetry group is $S U(5)_{L} \otimes S U(5)_{R}$ ?. A suitable field variation of the fifth flavour will then yield WIs concerning the operators of eqs. (12) and (16). Finally, since one of our aims is to propose WI's which can be used in simulations, we will be working with a small finite quark mass. It is to be understood, throughout the rest of this section, that the chiral limit is to be taken in the end.

We now establish our notation. For the purposes of this Section \pm superscripts will be dropped in what follows from operators and correlation functions (for example, from the $Q^{ \pm}$'s, $G^{ \pm}$'s and $\Lambda^{ \pm}$'s). We will be using Green functions which are expectation values of the following operators:

$$
\begin{aligned}
G_{k}\left(x_{0} ; x_{1}, x_{2}, x_{3}, x_{4}\right)_{\alpha \beta \gamma \delta}^{A B C D} & =\psi_{1}\left(x_{1}\right)_{\alpha}^{A} \bar{\psi}_{2}\left(x_{2}\right)_{\beta}^{B} Q_{k}\left(x_{0}\right) \psi_{3}\left(x_{3}\right)_{\gamma}^{C} \bar{\psi}_{4}\left(x_{4}\right)_{\delta}^{D} \\
\mathcal{G}_{k}\left(x_{0} ; x_{1}, x_{2}, x_{3}, x_{4}\right)_{\alpha \beta \gamma \delta}^{A B C D} & =\psi_{1}\left(x_{1}\right)_{\alpha}^{A} \bar{\psi}_{2}\left(x_{2}\right)_{\beta}^{B} \mathcal{Q}_{k}\left(x_{0}\right) \psi_{3}\left(x_{3}\right)_{\gamma}^{C} \bar{\psi}_{4}\left(x_{4}\right)_{\delta}^{D}
\end{aligned}
$$

for $k=1, \ldots, 5$. The operators $Q_{k}$ and $\mathcal{Q}_{k}$ on the r.h.s. are those of eqs. (12) and (16); i.e. they are defined in terms of fermion fields with flavours $1, \cdots, 4$. Their vacuum expectation values are the four-quark correlation functions of eq.(25), which are the starting point of the $R I$ renormalization procedure. We will also be using five other operators (and their four-quark correlation functions) which are defined as follows:

$$
\begin{aligned}
\breve{\mathcal{G}}_{k}\left(x_{0} ; x_{1}, x_{2}, x_{3}, x_{4}\right)_{\alpha \beta \gamma \delta}^{A B C D} & =\psi_{1}\left(x_{1}\right)_{\alpha}^{A} \bar{\psi}_{2}\left(x_{2}\right)_{\beta}^{B} \breve{\mathcal{Q}}_{k}\left(x_{0}\right) \psi_{3}\left(x_{3}\right)_{\gamma}^{C} \bar{\psi}_{4}\left(x_{4}\right)_{\delta}^{D} \\
\mathcal{G}_{k}^{5}\left(x_{0} ; x_{1}, x_{2}, x_{3}, x_{4}\right)_{\alpha \beta \gamma \delta}^{A B C D} & =\left[\gamma_{5} \psi_{5}\left(x_{1}\right)\right]_{\alpha}^{A} \bar{\psi}_{2}\left(x_{2}\right)_{\beta}^{B} \breve{\mathcal{Q}}_{k}\left(x_{0}\right) \psi_{3}\left(x_{3}\right)_{\gamma}^{C} \bar{\psi}_{4}\left(x_{4}\right)_{\delta}^{D}
\end{aligned}
$$

where

$$
\breve{\mathcal{Q}}_{k}^{ \pm}=\left(\bar{\psi}_{5} \Gamma^{(1)} \psi_{2}\right)\left(\bar{\psi}_{3} \Gamma^{(2)} \psi_{4}\right) \pm\left(\bar{\psi}_{5} \Gamma^{(1)} \psi_{4}\right)\left(\bar{\psi}_{3} \Gamma^{(2)} \psi_{2}\right)
$$

and $k=1, \cdots, 5$ stands for the combination of Dirac matrices corresponding to the definitions of eq. (16). In other words, $\breve{\mathcal{Q}}_{k}^{ \pm}$is obtained from $\mathcal{Q}_{k}^{ \pm}$by substituting $\bar{\psi}_{1}$

\footnotetext{
${ }^{9}$ In the end of this subsection, we will comment on the relevance of our results to the physical case of three light quark flavours.
} 
by $\bar{\psi}_{5}$. The operators $\mathcal{G}_{k}^{5}$ are obtained from $\mathcal{G}_{k}$, with the substitution $\left(\psi_{1}, \bar{\psi}_{1}\right) \rightarrow$ $\left(\gamma_{5} \psi_{5}, \bar{\psi}_{5}\right)$. The colour and spin indices $(A, B, \ldots$ and $\alpha, \beta, \ldots)$ will be dropped from now on for notational simplicity. We will also need the axial current and pseudoscalar density

$$
\begin{aligned}
A_{\mu}(x) & =\bar{\psi}_{1}(x) \gamma_{\mu} \gamma_{5} \psi_{5}(x) \\
P(x) & =\bar{\psi}_{1}(x) \gamma_{5} \psi_{5}(x)
\end{aligned}
$$

We perform infinitesimal axial transformations on quark fields in terms of the $S U(5)$ raising operator

$$
\lambda=\left(\begin{array}{lllll}
0 & 0 & 0 & 0 & 1 \\
0 & 0 & 0 & 0 & 0 \\
0 & 0 & 0 & 0 & 0 \\
0 & 0 & 0 & 0 & 0 \\
0 & 0 & 0 & 0 & 0
\end{array}\right)
$$

which induces transformations between the first and fifth flavours only:

$$
\begin{aligned}
& \psi_{1} \rightarrow \psi_{1}+i \delta \alpha \gamma_{5} \psi_{5} \\
& \bar{\psi}_{5} \rightarrow \bar{\psi}_{5}+i \delta \alpha \bar{\psi}_{1} \gamma_{5}
\end{aligned}
$$

Correspondingly, operators $\breve{\mathcal{Q}}$ transform into their $Q$ counterparts:

$$
\begin{aligned}
& \breve{\mathcal{Q}}_{1} \rightarrow \breve{\mathcal{Q}}_{1}-i \delta \alpha Q_{1} \\
& \breve{\mathcal{Q}}_{2} \rightarrow \breve{\mathcal{Q}}_{2}+i \delta \alpha Q_{2} \\
& \breve{\mathcal{Q}}_{3} \rightarrow \breve{\mathcal{Q}}_{3}+i \delta \alpha Q_{3} \\
& \breve{\mathcal{Q}}_{4} \rightarrow \breve{\mathcal{Q}}_{4}+i \delta \alpha Q_{4} \\
& \breve{\mathcal{Q}}_{5} \rightarrow \breve{\mathcal{Q}}_{5}+i \delta \alpha Q_{5}
\end{aligned}
$$

Whenever convenient, matrix notation will be used, as in subsect. 4.2. We define the $1 \times 5$ row vectors

$$
\begin{aligned}
\breve{\mathcal{G}} & =\left(\breve{\mathcal{G}}_{1}, \breve{\mathcal{G}}_{2}, \breve{\mathcal{G}}_{3}, \breve{\mathcal{G}}_{4}, \breve{\mathcal{G}}_{5}\right) \\
G & =\left(-G_{1}, G_{2}, G_{3}, G_{4}, G_{5}\right) \\
\mathcal{G}^{5} & =\left(\mathcal{G}_{1}^{5}, \mathcal{G}_{2}^{5}, \mathcal{G}_{3}^{5}, \mathcal{G}_{4}^{5}, \mathcal{G}_{5}^{5}\right)
\end{aligned}
$$

Note the minus sign in the element $G_{1}$ of vector $G$; it has been introduced in its definition in order to take into account the minus sign of the axial variation of $\breve{\mathcal{Q}}_{1}$; c.f. eq.(71).

The lattice WI arising from this axial variation of the $1 \times 5$ row vector $\langle\breve{\mathcal{G}}\rangle$ is

$$
\begin{array}{r}
\frac{\delta}{\delta \alpha(x)}\left\langle\breve{\mathcal{G}}\left(x_{0} ; x_{1}, x_{2}, x_{3}, x_{4}\right)\right\rangle=0 \Leftrightarrow \\
\left\langle\frac{\delta}{\delta \alpha(x)} \breve{\mathcal{G}}\left(x_{0} ; x_{1}, x_{2}, x_{3}, x_{4}\right)\right\rangle=\left\langle\breve{\mathcal{G}}\left(x_{0} ; x_{1}, x_{2}, x_{3}, x_{4}\right) \frac{\delta S}{\delta \alpha(x)}\right\rangle
\end{array}
$$


where all points $x_{0}, x_{1}, x_{2}, x_{3}$ and $x_{4}$ are kept separate. The variation of the operator $\breve{\mathcal{G}}$ receives a contribution $\langle G\rangle$ from the variation of $\breve{\mathcal{Q}}$ and a contribution from the variation of the fermion field $\psi_{1}$. Integrating over $x$ yields

$$
\langle G\rangle+\left\langle\mathcal{G}^{5}\right\rangle=-\int d^{4} x\left\langle\breve{G}\left[\nabla_{x}^{\mu} A_{\mu}(x)-2 m_{0} P(x)-X_{5}(x)\right]\right\rangle
$$

In the chiral limit, the term $2 m_{0} P(x)$ on the r.h.s. vanishes, whereas the integrated total divergence of the axial current gives a non-zero surface term, due to the presence of Goldstone bosons. If we are not in the chiral limit, the $2 m_{0} P(x)$ term is present, but the surface term from the current divergence vanishes upon integration. We will be considering the latter case, in order to mimic what is happening in the simulations (i.e. first we compute at small non-zero quark mass and then extrapolate to the chiral limit). The operator $X_{5}$ arises from the variation of the chiral symmetry breaking Wilson term in the action. As shown in ref. [11] (see also [17] for a detailed discussion) it mixes, under renormalization with $\nabla^{\mu} A_{\mu}$ and $P$. This mixing, determined by the requirement that on-shell matrix elements of the subtracted $X_{5}$ vanish in the continuum, generates a finite renormalization of the axial current and a power subtraction of the quark mass. Thus, following ref. [11], in the above WI we will trade-off $X_{5}$ for the renormalized expression $\left[\nabla^{\mu} \hat{A}_{\mu}-2 \hat{m} \hat{P}-\bar{X}_{5}\right]$, where $\bar{X}_{5}$ is the subtracted $X_{5}$. What is of interest to us is that, besides the above renormalizations, the $\bar{X}_{5}$ insertion in the above correlation function also generates contact terms. They are found by looking at the flavour content and discrete symmetries of the specific correlation function. We find (up to Schwinger terms which will vanish under the integral):

$$
\begin{aligned}
\left\langle\bar{X}_{5}(x) \breve{\mathcal{G}}_{k}\left(x_{0} ; x_{1}, x_{2}, x_{3}, x_{4}\right)\right\rangle & =\left\langle G_{j}\right\rangle\left(\delta_{j k}-R_{j k}\right) \delta\left(x-x_{0}\right) \\
& -\left\langle G_{j}\right\rangle \Delta_{i j} R_{i k} \delta\left(x-x_{0}\right)
\end{aligned}
$$

where repeated indices are summed; $i, j=1, \cdots, 5$. The notation for the various coefficients has been chosen with some foresight: The $5 \times 5$ matrix $R_{j k}$ is a block diagonal matrix of the form of $Z_{\chi}$ of eq. (22). It will eventually turn out that $R=Z_{\chi}^{T}\left(\mathcal{Z}^{-1}\right)^{T}$; i.e. $R$ is the finite ratio of the parity-conserving to parity-violating, logarithmically diverging, renormalization matrices of $\chi R S$ type. The $5 \times 5$ matrix $\Delta_{i j}$ is a sparse matrix of the form of eq. (23); it will eventually turn out to be the matrix defined in eq. (23). Finally note that the product $\left[\Delta^{T} R\right]_{j k}$ is a sparse matrix of the form of $\Delta$. In conclusion, we have separated the contact terms on the r.h.s. of eq. (75) into a first term which mixes the $\left\langle G_{j}\right\rangle$ 's as in the $\chi R S$ scheme and a second term which generates lattice mixing. Note that in the above expression, we have only included the contact terms generated by the proximity of $\bar{X}_{5}(x)$ to the operators $\mathcal{Q}\left(x_{0}\right)$. In principle there are also contact terms arising from the proximity of $\bar{X}_{5}(x)$ to the quark fields of the correlation $\mathcal{G}$ at points $x_{1}, \cdots, x_{4}$. However, as shown in ref. [42], these terms vanish in the continuum limit. The reason is that the insertion of the operator $\bar{X}_{5}$ with fundamental fields (once both this operator and the quark fields are renormalized) is proportional to the lattice spacing. 
We now combine eqs. (74) and (75), Fourier-transform the WI (with all external momenta set equal to $p$ ) and amputate the resulting correlation functions. We denote by $\Lambda(p)$ and $\mathcal{J}^{5}(p)$ the momentum-space amputated Green functions of $\langle G\rangle$ and $\left\langle\mathcal{G}^{5}\right\rangle$. The Fourier-transformed $\breve{\mathcal{G}}$ is denoted by $\breve{\mathcal{G}}(p)$. The resulting momentum-space amputated WI is

$$
\Lambda(p)\left[I+\Delta^{T}\right] R=-\mathcal{X}^{5}(p)+2 \hat{m} \int d^{4} x\langle\breve{\mathcal{G}}(p) \hat{P}(x)\rangle \prod_{1}^{4}\left\langle S^{-1}(p)\right\rangle
$$

We require that the above WI, up to quark field renormalization, be the one valid in the continuum limit for renormalized correlation functions (operators) and that it be identical to the corresponding nominal WI (which is just the tree-level version of the above equation). This implies that $\Delta$ is indeed the matrix of lattice subtractions. Moreover, $R$ can indeed be identified with the ratio of the renormalization matrices $Z_{\chi}^{T}\left(\mathcal{Z}^{-1}\right)^{T}$, since this combination would renormalize both sides of the above equation (NB: the renormalization of $\breve{\mathcal{G}}(p)$ is identical to that of $\mathcal{G}(p)$, since the two operators only differ by a relabelling of $\psi_{5}$ as $\psi_{1}$ ).

We now show how the WI fixes both the lattice mixing matrix $\Delta$ and the matrix ratio $R$. We consider the first column of the above WI; its l.h.s. concerns the operator $Q_{1}$ and its lattice mixings. By projecting this WI with five projectors of eqs. (37) and (38) we construct a linear inhomogenous system of five equations w.r.t. the quantities $R_{11}$ and $\left[\Delta^{T} R\right]_{k 1}=R_{11} \Delta_{1 k}$, with $k=2, \cdots, 5$. By solving this system, the lattice mixing of $Q_{1}$ and the ratio of the multiplicative renormalization constants $R_{11}=$ $Z_{11} / \mathcal{Z}_{11}$ of $Q_{1}$ and $\breve{\mathcal{Q}}_{1}$ (which is just $\mathcal{Q}_{1}$ with a flavour relabelling) can be determined. Similarly, a system of ten equations (using 10 projectors on the second and third columns of the WI) determines the lattice mixing of $Q_{2}$ and $Q_{3}$ (i.e. the $\Delta_{j k}$ 's with $j=2,3$ and $k=1,4,5)$ and the elements $R_{i k}$ with $i, k=2,3$. The case of $Q_{4}$ and $Q_{5}$ is identical to that of $Q_{2}$ and $Q_{3}$ (but involves the fourth and fifth rows of the WI).

So far we have shown how WIs can determine the lattice mixing coefficients and the finite ratio of the $\chi R S$ renormalization matrices. We will now show that these quantities are compatible to the ones obtained from the RI renormalization conditions, in the large momentum limit. We project both sides of eq. (76) with the $5 \times 1$ column vector $\mathrm{P}$, defined as

$$
\mathbf{P}=\left(-\mathbf{P}_{1}, \mathbf{P}_{2}, \mathbf{P}_{3}, \mathbf{P}_{4}, \mathbf{P}_{5}\right)^{T}
$$

(the minus sign in $\mathrm{P}_{1}$ corresponds to that of $-G_{1}$ in eq. (72). For large $p$, the last term on the r.h.s. vanishes. This is because the explicit $m_{0}$ factor implies that the integrated term has one less dimension that the others so that, at large momenta it vanishes faster by one power of $p$ (see ref. [42]). Moreover, in this limit the inverse quark propagator behaves as $S^{-1}(p)=i \Sigma_{1} \gamma_{\mu} p_{\mu}$ (with $\Sigma_{1}$ a scalar form factor and up to $\mathcal{O}(a)$ terms). This means that in this limit it anticommutes with $\gamma_{5}$. Combining this result with the definition of $\mathbf{P}_{k}$ we easily deduce that

$$
\operatorname{Tr} \mathrm{P}_{k} \mathcal{X}_{j}^{5}=-\operatorname{Tr} p_{k} \mathcal{\lambda}_{j}
$$


(with $k, j=1, \cdots, 5$ ). Thus, the WI becomes

$$
\operatorname{Tr} \mathrm{P} \cdot \Lambda\left[I+\Delta^{T}\right] Z_{\chi}^{T}=\operatorname{Tr} P \cdot \mathcal{N} \mathcal{Z}^{T}
$$

This WI, given the RI renormalization condition for the parity violating operator

$$
\operatorname{Tr} P \cdot \mathcal{\lambda} \mathcal{Z}^{T}=I
$$

implies the RI renormalization condition of eq. (56) for the parity conserving operators $Q$.

We now comment briefly on the relation of these results to the more realistic case of three light flavours. The physical operators $\mathrm{O}^{-}$always mix with others of lower dimension. This introduces further complications which are beyond the scope of the present work. For the physical operators $\mathrm{O}^{+}$, there are penguin-type contributions which, however, cancel. Thus, the resulting WIs are identical to the ones obtained here with five flavours.

\subsection{Scale dependence of the renormalization constants}

We will now specify which of these renormalization constants are divergent quantities in the UV limit and which are finite. First we give a general discussion of the functional dependence of the $Z$ 's (and $\mathcal{Z}$ 's) on the coupling, mass, renormalization point and cutoff. We are interested in the physics of light quark masses, so we assume $\mu \gg m$. The $Z$ 's are dimensionless quantities; thus, in principle, they could have the functional dependence $Z\left(g_{0}^{2}, a \mu, a m, m / \mu\right)$, where $m$ stands for the degenerate quark mass and $\mu$ is the renormalization scale (i.e. it stands for a generic choice of the four external quark momenta). It must be chosen so as to regularize all IR divergences, including those arising in the chiral limit. For the four-fermion operators we are considering in this work, it suffices to simply take equal momenta; i.e. $p_{i}^{2}=\mu^{2}(i=1, \ldots, 4$ for the four external legs; $\mu$ is space-like.). Any regular dependence on am and $a \mu$ should drop out in the continuum limit $a \rightarrow 0$, and is therefore treated in simulations as a systematic error due to the finiteness of the cutoff. These errors are, say, $\mathcal{O}(a m), \mathcal{O}(a \mu)$ for the Wilson action and $\mathcal{O}\left(a m g_{0}^{2}\right), \mathcal{O}\left(a \mu g_{0}^{2}\right)$ for the tree level improved Clover action, used in the present paper. Moreover, the $Z$ 's cannot be singular in am (e.g. have $\ln (a m)$ terms in the small mass limit $m \ll \mu$ ) because they would diverge not only in the continuum limit, but also in the chiral limit $(m \rightarrow 0)$. Similarly, regular terms in $m / \mu$ are neglected (light masses), whereas singular ones must be absent (existence of chiral limit). In conclusion, for light quark masses, the functional dependence of the $Z$ 's is in general of the form $Z\left(g_{0}^{2}, a \mu\right)$.

Power counting suggest that their dependence on $a \mu$ is singular (logarithmic). However, this is only true of the $\chi R S$ renormalization constants $Z_{\chi}$ and $\mathcal{Z}$. The lattice mixing coefficients $\Delta$ and the "ratio" $\mathcal{Z}^{-1} Z_{\chi}$ can be determined from a system of equations (the projected WI's discussed above). This implies that they can be expressed in 
terms of bare lattice correlation functions, which do not depend on the renormalization scale. Thus their functional dependence is of the form $Z\left(g_{0}^{2}\right)$; i.e. they are finite.

Finally, we can lift the mass degeneracy, introducing small mass differences $\delta m$. This could in principle introduce dependences like powers of $a \delta m$ and $\ln \left(m_{i} / m_{j}\right)$ in the $Z$ 's. However, as pointed out in [41], none of these survive: The regular terms (e.g. powers of $a \delta m$ ) vanish in the continuum limit $a \rightarrow 0$; i.e. they are part of the usual $O(a m)$ and $O\left(g_{0}^{2} a m\right)$ discretization errors. Singular (power or logarithmic) dependence is not allowed by the requirement that the chiral limit of the theory $\left(m_{i} \rightarrow 0\right)$ exist and it be well defined. Thus, lifting the mass degeneracy does not spoil the renormalization pattern discussed above.

\subsection{Identities between renormalization constants}

In this section we derive useful identities which relate some parity conserving renormalization constants of $\mathcal{S}=1$ operators to renormalization constants of their $\mathcal{S}=-1$ counterparts. These identities are formally exact, but are only approximately satisfied in practical computations. Thus, they are useful tests of the reliability of our results.

In order to derive them, we first need to show that, once the operator basis has been renormalized using the RI scheme, any other basis of renormalized operators (formed by linear combinations of the original renormalized operators) also satisfies the RI conditions. This is very straightforward. In the first basis, the RI scheme consists in the following properties of the projected amputated Green functions $\Lambda$ :

$$
\begin{aligned}
\operatorname{Tr} \mathrm{P} \Lambda^{(0)} & =I \\
\hat{\Lambda} & \equiv Z \Lambda \\
\operatorname{Tr} \mathrm{P} \hat{\Lambda} & =\operatorname{Tr} \mathrm{P}(Z \Lambda)=I
\end{aligned}
$$

The above are valid at the renormalization scale $p^{2}=\mu^{2}$. This shorthand notation should be clear to the reader. Now we can define a new basis of operators, obtained by a rotation $R$ of the original basis. This implies rotated projected amputated Green functions $\Lambda^{\prime}$ and rotated projectors $\mathbf{P}^{\prime}$, which must satisfy:

$$
\begin{aligned}
\Lambda^{\prime} & \equiv R \Lambda \\
\operatorname{Tr} \mathrm{P}^{\prime} \Lambda^{(0)^{\prime}} & =I
\end{aligned}
$$

Trivially, the last equation is satisfied, provided that

$$
\mathrm{P}^{\prime}=\mathrm{P} R^{-1}
$$

It is straightforward to combine eqs. 811)-(83) to show that also $\hat{\Lambda}^{\prime}$ satisfies the RI renormalization condition $\operatorname{Tr} \mathrm{P}^{\prime} \hat{\Lambda}^{\prime}=I$. The implication of this property is that if we use the RI scheme in order to renormalize separately the operators $O_{[V V+A A]}$ and $O_{[V V+A A]}^{F}$, for example, the renormalized operator $\hat{Q}_{[V V+A A]}+\hat{Q}_{[V V+A A]}^{F}$ satisfies the 
RI scheme; in other words it is the renormalized operator $\hat{Q}_{1}^{+}$. In this section, we will make use of this property. We also note that in this section we will exclusively work with the lattice subtracted parity conserving operators $\tilde{Q}_{k}$ and the parity violating operators $\mathcal{Q}_{k}$. As discussed in subsecs. 3.4 and 5.1, these operators have good chiral properties (in other words, they can be thought of as operators in the $\chi R S$ ).

We consider the operator $O_{[V A-A V]}$, which only mixes with the operator $O_{[S P-P S]}^{F}$. Thus, we renormalize it in the RI scheme to obtain

$$
\hat{O}_{[V A-A V]}=z_{22} O_{[V A-A V]}-z_{23} O_{[S P-P S]}^{F}
$$

The same renormalization pattern is obeyed by operator $O_{[V A-A V]}^{F}$ (it only mixes with $\left.O_{[S P-P S]}\right)$ since it only involves a relabelling of flavours:

$$
\hat{O}_{[V A-A V]}^{F}=z_{22} O_{[V A-A V]}^{F}-z_{23} O_{[S P-P S]}
$$

The last two equations can be combined into

$$
\hat{\mathcal{Q}}_{2}^{ \pm}=\hat{O}_{[V A-A V]} \pm \hat{O}_{[V A-A V]}^{F}=z_{22} \mathcal{Q}_{2}^{ \pm} \pm z_{23} \mathcal{Q}_{3}^{ \pm}
$$

which implies that

$$
\begin{aligned}
& \mathcal{Z}_{22}^{+}=\mathcal{Z}_{22}^{-}=z_{22} \\
& \mathcal{Z}_{23}^{+}=-\mathcal{Z}_{23}^{-}=z_{23}
\end{aligned}
$$

Similar expressions can be derived for the renormalization constants of operators $\mathcal{Q}_{3}^{ \pm}$ and the parity conserving ones in the $\chi R S$ scheme. In practical simulations, we expect that these identities are well satisfied in the parity violating case. For the parity conserving one (which also involves lattice subtractions) the agreement should only be approximate.

We note the above proof rests onto two crucial properties of the operators $O_{[V A-A V]}$ and $O_{[S P-P S]}^{F}$ concerned: (i) they mix with each other only; (ii) they transform into each other under Fierz transformations in Dirac space (cf. eq.(96)). These two properties determine the mixing pattern of eqs. (84) and (85). This proof cannot be extended to operators $\mathcal{Q}_{4}^{ \pm}$and $\mathcal{Q}_{5}^{ \pm}$. The second requirement is satisfied by the pair of operators $O_{[S P+P S]}$ and $O_{[S P+P S]}^{F}-O_{[T \tilde{T}]}^{F}$; see eq. (96). However, the first requirement is not satisfied, since operator $O_{[S P+P S]}$ mixes not only with $O_{[S P+P S]}^{F}-O_{[T \tilde{T}]}^{F}$, but also with $O_{[S P+P S]}-O_{[T \tilde{T}]}$ (cf. Table 1). Thus, no property analogous to eq. (87) can be found for the operators $\mathcal{Q}_{4}^{ \pm}$and $\mathcal{Q}_{5}^{ \pm}$(or a linear combination of them).

\section{$6 \quad$ Numerical results}

In order to test the feasibility of these ideas, we have computed the renormalization constants, in the RI scheme, of the complete basis of operators $Q_{k}$ and $\mathcal{Q}_{k}$, for $k=$ 
$1, \cdots, 5$. This was done in the quenched approximation, for the Wilson and treelevel improved Clover action. We have performed simulations at three values of the gauge coupling, namely $\beta=6 / g_{0}^{2}=6.0,6.2,6.4$. Our results were obtained at finite quark masses and extrapolated to the chiral limit $\kappa_{c}$. The specific values of all lattice parameters used in these simulations can be found in tab. 3. We also give in the table

\begin{tabular}{||l||c|c|c|c|c|c||}
\hline \hline$\beta$ & 6.0 & 6.0 & 6.2 & 6.2 & 6.4 & 6.4 \\
\hline Action & $\mathrm{C}$ & $\mathrm{W}$ & $\mathrm{C}$ & $\mathrm{W}$ & $\mathrm{C}$ & $\mathrm{W}$ \\
\# Confs & 100 & 100 & 180 & 100 & 60 & 60 \\
Volume & $16^{3} \times 32$ & $16^{3} \times 32$ & $16^{3} \times 32$ & $16^{3} \times 32$ & $24^{3} \times 32$ & $24^{3} \times 32$ \\
\hline$\kappa$ & 0.1425 & 0.1530 & 0.14144 & 0.1510 & 0.1400 & 0.1488 \\
& 0.1432 & 0.1540 & 0.14184 & 0.1515 & 0.1403 & 0.1492 \\
& 0.1440 & 0.1550 & 0.14224 & 0.1520 & 0.1406 & 0.1496 \\
& & & 0.14264 & 0.1526 & 0.1409 & 0.1500 \\
\hline$\kappa_{c}$ & 0.14551 & 0.15683 & 0.14319 & 0.15337 & 0.14143 & 0.15058 \\
\hline \hline$a^{-1}$ & $2.16(4)$ & $2.26(5)$ & $2.70(10)$ & $3.00(9)$ & $4.00(20)$ & $4.10(20)$ \\
\hline \hline
\end{tabular}

Table 3: Parameters of the runs used for the NP calculation of the renormalization constants. We also give the critical value of the hopping parameter $\kappa_{c}$ and the inverse lattice spacing $a^{-1}$, as quoted in ref. [43].

the value of the inverse lattice spacing $a^{-1}$, obtained in ref. 43 on the same dataset. All statistical errors have been estimated with the jacknife method, decimating 10 configurations at a time.

As has been explained in sec. 4, the NP method is based on the computation of quark Green's functions; thus gauge-fixing has to be implemented. We have worked in the lattice Landau gauge, defined by minimizing the functional

$$
\operatorname{Tr}\left[\sum_{\mu=1}^{4}\left(U_{\mu}(x)+U_{\mu}^{\dagger}(x)\right)\right] .
$$

Possible effects from Gribov copies have been ignored. However, in analogy to the study of the effect of Gribov ambiguities on the renormalization of two-quark operators of ref. [15], we expect them to be small.

We now present detailed results for one representative case, namely the renormalization constants of the Clover action at $\beta=6.2$, as a function of the renormalization scale in lattice units. All results are extrapolated in the chiral limit. In Figs. I we plot the renormalization constants of operators $Q_{1}^{+}$and $\mathcal{Q}_{1}^{+}$. We see that both $\chi R S$ renormalization constants $Z_{11}^{+}$and $\mathcal{Z}_{11}^{+}$are scale dependent, whereas the mixing coefficients $\Delta_{1 k}(k=2, \ldots 5)$ become more stable with increasing $\mu$. This is what we 
expect from the discussion of sect. 5. In figs. 2 and 3 we show similar results for the renormalization constants of the operators $Q_{k}^{+}$and $\mathcal{Q}_{k}^{+}$, for $k=2,3$, whereas in figs. 4 and 5 we show those for $k=4,5$. Most $\chi \mathrm{RS}$ renormalization constants of the matrix

$Z_{\chi}^{+}$display a marked $\mu$-dependence due to a non-zero anomalous dimension, whereas the lattice subtraction coefficients of the matrix $\Delta^{+}$are roughly scale-independent in the window $a \mu \in[1,2]$. Compared to the others, the renormalization constants $\Delta_{24}^{+}$, $\Delta_{34}^{+}$and $\Delta_{43}^{+}$have a more pronounced variation with the scale $\mu$; nevertheless they are reasonably flat in the same window.

In order to give a more quantitative flair of our results, we present the renormalization matrices, for all actions and couplings, in Appendix G. Only results at the renormalization scale $\mu \simeq 2 \mathrm{GeV}$ are presented. Note that the identities derived in subsec. 5.3 for the $\chi R S$ renormalization constants of operators $\mathcal{Q}_{2}^{ \pm}$and $\mathcal{Q}_{3}^{ \pm}$(c.f. eq.(87)) are well satisfied. For the parity conserving operators $Q_{2}^{ \pm}$and $Q_{3}^{ \pm}$similar identities appear to be approximately true. At the scale $\mu \simeq 2 \mathrm{GeV}$, these results can be directly used in the computation of the matrix elements of the corresponding operators. A first implementation of these results in the calculation of various $B$-parameters can be found in refs. [29, 30].

The validity of our results should be confirmed independently from WIs, computed with the same actions and at the same couplings. Work in this direction is in progress.

\section{Acknowledgements}

We warmly thank E. Franco, L. Giusti, G.C. Rossi and M. Testa for many stimulating and thorough discussions. A careful reading of the manuscript by G.C. Rossi is also gratefully acknowledged. V.G. acknowledges the partial support by CICYT under grant number AEN-96/1718.

\section{A Notation and conventions}

The 16 Euclidean Dirac $4 \times 4$ matrices, which form a complete basis, are denoted by

$$
\Gamma=\left\{\mathbf{I}, \gamma_{\mu}, \sigma_{\mu \nu}, \gamma_{\mu} \gamma_{5}, \gamma_{5}\right\} \equiv\{S, V, T, A, P\}
$$

where

$$
\begin{aligned}
& \gamma_{5} \equiv-\frac{1}{4 !} \epsilon_{\mu \nu \rho \lambda} \gamma_{\mu} \gamma_{\nu} \gamma_{\rho} \gamma_{\lambda}=-\gamma_{0} \gamma_{1} \gamma_{2} \gamma_{3} \\
& \sigma_{\mu \nu}=\frac{1}{2}\left[\gamma_{\mu}, \gamma_{\nu}\right]
\end{aligned}
$$

with $\epsilon_{\mu \nu \rho \lambda}$ the completely antisymmetric rank- four pseudotensor with $\epsilon_{0123}=+1$. The Euclidean Dirac matrices satisfy the following properties

$$
\left\{\gamma_{\mu}, \gamma_{\nu}\right\}=2 \delta_{\mu \nu}, \quad \gamma_{\mu}^{\dagger}=\gamma_{\mu}, \quad \gamma_{5}^{\dagger}=\gamma_{5}
$$


We also define the dual "sigma" matrix

$$
\tilde{\sigma}_{\mu \nu} \equiv \frac{1}{2} \epsilon_{\mu \nu \rho \lambda} \sigma_{\rho \lambda}=\gamma_{5} \sigma_{\mu \nu} \equiv \tilde{T}
$$

The helicity projectors are, as usual,

$$
\gamma_{\mu}^{L} \equiv L_{\mu} \equiv \gamma_{\mu}\left(1-\gamma_{5}\right), \quad \gamma_{\mu}^{R} \equiv R_{\mu} \equiv \gamma_{\mu}\left(1+\gamma_{5}\right)
$$

and

$$
L \equiv\left(1-\gamma_{5}\right), \quad R \equiv\left(1+\gamma_{5}\right) .
$$

Repeated $\Gamma$ matrices imply summation of their Lorentz indices (if any); for example $V V \equiv \sum_{\mu} \gamma_{\mu} \otimes \gamma_{\mu}, V A \equiv \sum_{\mu} \gamma_{\mu} \otimes \gamma_{\mu} \gamma_{5}$, etc. Note however, that $T T=\sigma_{\mu \nu} \otimes \sigma_{\mu \nu}$ and $T \tilde{T}=\sigma_{\mu \nu} \otimes \tilde{\sigma}_{\mu \nu}$ means summation over the 6 independent $\sigma_{\mu \nu}$ matrices (e.g. summation over $\mu$ and $\nu$ with $\mu>\nu)$.

The colour group is $S U\left(N_{c}\right)$ with $N_{c}=3$. The Gell- Mann group generators are denoted by $t^{a}, a=1, \ldots, N_{c}^{2}-1$. Fermion fields $\psi^{A \alpha}$ carry spinor and colour indices. Latin uppercase letters denote colour indices in the fundamental representation $\left(A, B, \ldots=1, \ldots, N_{c}\right)$, whereas Greek lowercase letters stand for Dirac spinor indices $(\alpha, \beta, \ldots, \rho, \sigma, \ldots=1, \ldots, 4)$. The letters $m$ and $n$ are reserved for Lorentz indices running on the set of Dirac matrices (as in $\Gamma^{(1) m} \Gamma^{(2) m}$ ) according to the following convention: if we are dealing with pairs of Dirac matrices such as $S S, S P$ etc., the index $m$ is absent. If we have pairs like $V V, V A$ etc, $m$ runs over the four values of $\gamma_{\mu}$. In the case of the pairs $T T$ and $T \tilde{T}, m$ runs over the six independent values of the Dirac matrices $\sigma_{\mu \nu}$ and $\tilde{\sigma}_{\mu \nu}$.

\section{B Fierz transformations in Dirac space}

In this Appendix we gather several useful formulæ concerning Fierz transformations in Dirac space; colour indices are ignored. We express the Fierz transformation of the Dirac indices of a four fermion operator as follows

$$
\boldsymbol{\Gamma} \otimes \boldsymbol{\Gamma} \equiv \Gamma_{\alpha \beta} \otimes \Gamma_{\gamma \delta} \rightarrow[\boldsymbol{\Gamma} \otimes \boldsymbol{\Gamma}]^{F_{D}} \equiv \Gamma_{\alpha \delta} \otimes \Gamma_{\gamma \beta}
$$

The Euclidean Fierz transformed Dirac tensor products $[\boldsymbol{\Gamma} \otimes \boldsymbol{\Gamma}]^{F_{D}}$ can be reexpressed as a linear combination of the complete set of the original tensor products $\boldsymbol{\Gamma} \otimes \boldsymbol{\Gamma}$, by exploiting the completeness of the set of Dirac matrices. One has

$$
\left(\begin{array}{c}
{[\boldsymbol{S} \otimes \tilde{\boldsymbol{S}}]^{F_{D}}} \\
{[\boldsymbol{V} \otimes \tilde{\boldsymbol{V}}]^{F_{D}}} \\
{[\boldsymbol{T} \otimes \tilde{\boldsymbol{T}}]^{F_{D}}} \\
{[\boldsymbol{A} \otimes \tilde{\boldsymbol{A}}]^{F_{D}}} \\
{[\boldsymbol{P} \otimes \tilde{\boldsymbol{P}}]^{F_{D}}}
\end{array}\right)=-\frac{1}{4}\left(\begin{array}{rrrrr}
1 & 1 & -1 & -1 & 1 \\
4 & -2 & 0 & -2 & -4 \\
-6 & 0 & -2 & 0 & -6 \\
-4 & -2 & 0 & -2 & 4 \\
1 & -1 & -1 & 1 & 1
\end{array}\right)\left(\begin{array}{c}
\boldsymbol{S} \otimes \boldsymbol{S} \\
\boldsymbol{V} \otimes \boldsymbol{V} \\
\boldsymbol{T} \otimes \boldsymbol{T} \\
\boldsymbol{A} \otimes \boldsymbol{A} \\
\boldsymbol{P} \otimes \boldsymbol{P}
\end{array}\right)
$$


The overall minus sign is due to the anticommutativity of the Fermi fields. Our Dirac $\Gamma$ matrices are normalized as

$$
S S=1, V V=4, T T=-6, A A=-4, P P=1
$$

with the summation over Dirac indices understood. Recall that the sum in $T T$ runs only over the six independent $\sigma_{\mu \nu}$ matrices $\square$.

From the above equation, we can easily derive the following useful identities, concerning parity conserving operators:

$$
\begin{array}{lll}
{[\boldsymbol{V} \otimes \boldsymbol{V}+\boldsymbol{A} \otimes \boldsymbol{A}]^{F_{D}}} & =\boldsymbol{V} \otimes \boldsymbol{V}+\boldsymbol{A} \otimes \boldsymbol{A} \\
{[\boldsymbol{V} \otimes \boldsymbol{V}-\boldsymbol{A} \otimes \boldsymbol{A}]^{F_{D}}} & = & -2[\boldsymbol{S} \otimes \boldsymbol{S}-\boldsymbol{P} \otimes \boldsymbol{S}] \\
{[\boldsymbol{S} \otimes \boldsymbol{S}-\boldsymbol{P} \otimes \boldsymbol{P}]^{F_{D}}} & =-\frac{1}{2}[\boldsymbol{V} \otimes \boldsymbol{V}-\boldsymbol{A} \otimes \boldsymbol{A}] \\
{[\boldsymbol{S} \otimes \boldsymbol{S}+\boldsymbol{P} \otimes \boldsymbol{P}]^{F_{D}}} & =-\frac{1}{2}[\boldsymbol{S} \otimes \boldsymbol{S}+\boldsymbol{P} \otimes \boldsymbol{P}-\boldsymbol{T} \otimes \boldsymbol{T}] \\
{[\boldsymbol{S} \otimes \boldsymbol{S}+\boldsymbol{P} \otimes \boldsymbol{P}-\boldsymbol{T} \otimes \boldsymbol{T}]^{F_{D}}} & =-2[\boldsymbol{S} \otimes \boldsymbol{S}+\boldsymbol{P} \otimes \boldsymbol{P}]
\end{array}
$$

Moreover, by substituting $\psi_{4} \rightarrow \gamma_{5} \psi_{4}$ in the above equations, we derive, for the parity violating operators $\mathcal{Q}_{1}^{ \pm}, \mathcal{Q}_{2}^{ \pm}$and $\mathcal{Q}_{3}^{ \pm}$of our basis:

$$
\begin{array}{lll}
{[\boldsymbol{V} \otimes \boldsymbol{A}+\boldsymbol{A} \otimes \boldsymbol{V}]^{F_{D}}} & =\boldsymbol{V} \otimes \boldsymbol{A}+\boldsymbol{A} \otimes \boldsymbol{V} \\
{[\boldsymbol{V} \otimes \boldsymbol{A}-\boldsymbol{A} \otimes \boldsymbol{V}]^{F_{D}}} & = & -2[\boldsymbol{S} \otimes \boldsymbol{P}-\boldsymbol{P} \otimes \boldsymbol{S}] \\
{[\boldsymbol{S} \otimes \boldsymbol{P}-\boldsymbol{P} \otimes \boldsymbol{S}]^{F_{D}}} & = & -\frac{1}{2}[\boldsymbol{V} \otimes \boldsymbol{A}-\boldsymbol{A} \otimes \boldsymbol{V}] \\
{[\boldsymbol{S} \otimes \boldsymbol{P}+\boldsymbol{P} \otimes \boldsymbol{S}]^{F_{D}}} & = & -\frac{1}{2}[\boldsymbol{S} \otimes \boldsymbol{P}+\boldsymbol{P} \otimes \boldsymbol{S}-\boldsymbol{T} \otimes \tilde{\boldsymbol{T}}] \\
{[\boldsymbol{S} \otimes \boldsymbol{P}+\boldsymbol{P} \otimes \boldsymbol{S}-\boldsymbol{T} \otimes \tilde{\boldsymbol{T}}]^{F_{D}}} & = & -2[\boldsymbol{S} \otimes \boldsymbol{S}+\boldsymbol{P} \otimes \boldsymbol{P}]
\end{array}
$$

Finally, from eq. (94) we can easily derive the five linear combinations of operators which are eigenstates of the transformation with eigenvalues \pm 1 ; they are:

$$
\begin{aligned}
{[\boldsymbol{S} \otimes \boldsymbol{S}+\boldsymbol{P} \otimes \boldsymbol{P}+\boldsymbol{T} \otimes \boldsymbol{T}]^{F_{D}} } & =+[\boldsymbol{S} \otimes \boldsymbol{S}+\boldsymbol{P} \otimes \boldsymbol{P}+\boldsymbol{T} \otimes \boldsymbol{T}] \\
{[\boldsymbol{V} \otimes \boldsymbol{V}+\boldsymbol{A} \otimes \boldsymbol{A}]^{F_{D}} } & =+[\boldsymbol{V} \otimes \boldsymbol{V}+\boldsymbol{A} \otimes \boldsymbol{A}] \\
{[2(\boldsymbol{S} \otimes \boldsymbol{S}-\boldsymbol{P} \otimes \boldsymbol{P})-(\boldsymbol{V} \otimes \boldsymbol{V}-\boldsymbol{A} \otimes \boldsymbol{A})]^{F_{D}} } & =+[2(\boldsymbol{S} \otimes \boldsymbol{S}-\boldsymbol{P} \otimes \boldsymbol{P})-(\boldsymbol{V} \otimes \boldsymbol{V}-\boldsymbol{A} \otimes \boldsymbol{A})] \\
{[2(\boldsymbol{S} \otimes \boldsymbol{S}-\boldsymbol{P} \otimes \boldsymbol{P})+(\boldsymbol{V} \otimes \boldsymbol{V}-\boldsymbol{A} \otimes \boldsymbol{A})]^{F_{D}} } & =-[2(\boldsymbol{S} \otimes \boldsymbol{S}-\boldsymbol{P} \otimes \boldsymbol{P})+(\boldsymbol{V} \otimes \boldsymbol{V}-\boldsymbol{A} \otimes \boldsymbol{A})] \\
{\left[\boldsymbol{S} \otimes \boldsymbol{S}+\boldsymbol{P} \otimes \boldsymbol{P}-\frac{1}{3} \boldsymbol{T} \otimes \boldsymbol{T}\right]^{F_{D}} } & =-\left[\boldsymbol{S} \otimes \boldsymbol{S}+\boldsymbol{P} \otimes \boldsymbol{P}-\frac{1}{3} \boldsymbol{T} \otimes \boldsymbol{T}\right]
\end{aligned}
$$

Thus, in Dirac space, three linear combinations are Fierz eigenstates with eigenvalue +1 and two are Fierz eigenstates with eigenvalue -1 .

\footnotetext{
${ }^{10}$ Sometimes $T$ and $A$ are defined with an imaginary $i$ prefactor in order to have a positive normalization. This would imply a change of sign in the $T$ and $A$ rows and columns of the $F$ matrix. This convention is not adopted here.
} 


\section{Numerical results}

In this appendix we present the renormalization matrices for both the Wilson and Clover action at the three couplings considered. All results are in the chiral limit. For each $\beta$ value, we show results at a scale $a \mu$ (in lattice units) such that $\mu \simeq 2 \mathrm{GeV}$.

C.1 Clover action, $\beta=6.0, \mu^{2} a^{2}=0.964$

$$
\begin{aligned}
& \mathcal{Z}^{-}=\left(\begin{array}{rrrrr}
0.90(4) & 0 & 0 & 0 & 0 \\
0 & 0.97(3) & -0.45(1) & 0 & 0 \\
0 & -0.017(3) & 0.36(1) & 0 & 0 \\
0 & 0 & 0 & 0.27(1) & -0.024(6) \\
0 & 0 & 0 & 0.23(1) & 1.12(4)
\end{array}\right) \\
& Z_{\chi}^{-}=\left(\begin{array}{rrrrr}
0.92(4) & 0 & 0 & 0 & 0 \\
0 & 0.99(3) & -0.42(1) & 0 & 0 \\
0 & -0.019(4) & 0.47(1) & 0 & 0 \\
0 & 0 & 0 & 0.36(2) & -0.024(6) \\
0 & 0 & 0 & 0.19(1) & 1.11(4)
\end{array}\right) \\
& \Delta^{-}=\left(\begin{array}{rrrrr}
0 & -0.15(1) & -0.02(1) & -0.15(2) & -0.09(1) \\
-0.23(1) & 0 & 0 & 0.38(2) & 0.06(1) \\
-0.059(5) & 0 & 0 & 0.53(1) & -0.019(8) \\
-0.06(1) & -0.02(1) & 0.79(2) & 0 & 0 \\
-0.053(8) & 0.048(6) & -0.300(9) & 0 & 0
\end{array}\right) \\
& \mathcal{Z}^{+}=\left(\begin{array}{rrrrr}
0.87(2) & 0 & 0 & 0 & 0 \\
0 & 0.96(3) & 0.45(1) & 0 & 0 \\
0 & 0.016(3) & 0.35(1) & 0 & 0 \\
0 & 0 & 0 & 0.44(1) & -0.009(3) \\
0 & 0 & 0 & -0.44(2) & 1.20(3)
\end{array}\right) \\
& Z_{\chi}^{+}=\left(\begin{array}{rrrrr}
0.88(2) & 0 & 0 & 0 & 0 \\
0 & 0.97(3) & 0.38(1) & 0 & 0 \\
0 & 0.010(3) & 0.41(1) & 0 & 0 \\
0 & 0 & 0 & 0.52(2) & -0.009(4) \\
0 & 0 & 0 & -0.41(2) & 1.20(4)
\end{array}\right) \\
& \Delta^{+}=\left(\begin{array}{rrrrr}
0 & -0.27(1) & -0.022(7) & 0.14(1) & 0.034(8) \\
-0.203(9) & 0 & 0 & -0.49(2) & 0.02(1) \\
0.041(2) & 0 & 0 & 0.70(2) & 0.001(7) \\
0.029(2) & -0.010(4) & 0.46(1) & 0 & 0 \\
0.037(5) & 0.012(5) & 0.273(9) & 0 & 0
\end{array}\right)
\end{aligned}
$$


C.2 Wilson action, $\beta=6.0, \mu^{2} a^{2}=0.964$

$$
\begin{aligned}
& \mathcal{Z}^{-}=\left(\begin{array}{rrrrr}
0.651(7) & 0 & 0 & 0 & 0 \\
0 & 0.611(9) & -0.262(5) & 0 & 0 \\
0 & -0.018(1) & 0.316(7) & 0 & 0 \\
0 & 0 & 0 & 0.271(8) & 0.007(2) \\
0 & 0 & 0 & 0.178(4) & 0.721(9)
\end{array}\right) \\
& Z_{\chi}^{-}=\left(\begin{array}{rrrrr}
0.655(8) & 0 & 0 & 0 & 0 \\
0 & 0.620(9) & -0.253(4) & 0 & 0 \\
0 & -0.021(2) & 0.35(1) & 0 & 0 \\
0 & 0 & 0 & 0.294(9) & 0.008(2) \\
0 & 0 & 0 & 0.166(5) & 0.72(1)
\end{array}\right) \\
& \Delta^{-}=\left(\begin{array}{rrrrr}
0 & -0.092(3) & 0.014(6) & -0.058(7) & -0.040(7) \\
-0.152(8) & 0 & 0 & 0.259(9) & 0.022(6) \\
-0.034(4) & 0 & 0 & 0.36(1) & 0.011(4) \\
-0.021(4) & -0.023(3) & 0.50(1) & 0 & 0 \\
-0.024(3) & 0.017(3) & -0.194(6) & 0 & 0
\end{array}\right) \\
& \mathcal{Z}^{+}=\left(\begin{array}{rrrrr}
0.532(7) & 0 & 0 & 0 & 0 \\
0 & 0.611(8) & 0.262(5) & 0 & 0 \\
0 & 0.018(1) & 0.316(8) & 0 & 0 \\
0 & 0 & 0 & 0.363(7) & -0.015(2) \\
0 & 0 & 0 & -0.239(5) & 0.678(9)
\end{array}\right) \\
& Z_{\chi}^{+}=\left(\begin{array}{rrrrr}
0.538(8) & 0 & 0 & 0 & 0 \\
0 & 0.610(9) & 0.241(6) & 0 & 0 \\
0 & 0.017(1) & 0.330(8) & 0 & 0 \\
0 & 0 & 0 & 0.388(8) & -0.016(2) \\
0 & 0 & 0 & -0.227(5) & 0.678(9)
\end{array}\right) \\
& \Delta^{+}=\left(\begin{array}{rrrrr}
0 & -0.176(8) & -0.031(5) & 0.054(6) & 0.007(4) \\
-0.122(6) & 0 & 0 & -0.30(1) & 0.011(4) \\
0.025(1) & 0 & 0 & 0.44(1) & -0.013(2) \\
0.011(1) & 0.009(2) & 0.308(9) & 0 & 0 \\
0.013(2) & 0.010(2) & 0.186(5) & 0 & 0
\end{array}\right)
\end{aligned}
$$

C.3 Clover action, $\beta=6.2, \mu^{2} a^{2}=0.617$

$$
\mathcal{Z}^{-}=\left(\begin{array}{rrrrr}
0.97(2) & 0 & 0 & 0 & 0 \\
0 & 1.04(2) & -0.51(1) & 0 & 0 \\
0 & -0.016(2) & 0.337(9) & 0 & 0 \\
0 & 0 & 0 & 0.28(1) & -0.029(8) \\
0 & 0 & 0 & 0.27(1) & 1.21(3)
\end{array}\right)
$$




$$
\begin{aligned}
& Z_{\chi}^{-}=\left(\begin{array}{rrrrr}
0.98(3) & 0 & 0 & 0 & 0 \\
0 & 1.07(2) & -0.49(2) & 0 & 0 \\
0 & -0.025(3) & 0.48(2) & 0 & 0 \\
0 & 0 & 0 & 0.32(1) & -0.017(6) \\
0 & 0 & 0 & 0.21(1) & 1.24(3)
\end{array}\right) \\
& \Delta^{-}=\left(\begin{array}{rrrrr}
0 & -0.14(1) & -0.03(2) & -0.16(1) & -0.11(1) \\
-0.20(1) & 0 & 0 & 0.37(2) & 0.060(9) \\
-0.054(9) & 0 & 0 & 0.52(3) & -0.008(9) \\
-0.060(8) & -0.02(1) & 0.77(3) & 0 & 0 \\
-0.072(4) & 0.042(5) & -0.294(9) & 0 & 0
\end{array}\right) \\
& \mathcal{Z}^{+}=\left(\begin{array}{rrrrr}
0.94(2) & 0 & 0 & 0 & 0 \\
0 & 1.04(2) & 0.51(1) & 0 & 0 \\
0 & 0.017(2) & 0.338(9) & 0 & 0 \\
0 & 0 & 0 & 0.44(1) & -0.012(3) \\
0 & 0 & 0 & -0.52(1) & 1.36(2)
\end{array}\right) \\
& Z_{\chi}^{+}=\left(\begin{array}{rrrrr}
0.96(2) & 0 & 0 & 0 & 0 \\
0 & 1.03(2) & 0.41(1) & 0 & 0 \\
0 & 0.007(3) & 0.41(2) & 0 & 0 \\
0 & 0 & 0 & 0.54(2) & -0.007(4) \\
0 & 0 & 0 & -0.47(1) & 1.35(2)
\end{array}\right) \\
& \Delta^{+}=\left(\begin{array}{rrrrr}
0 & -0.25(1) & -0.01(1) & 0.12(1) & 0.040(9) \\
-0.200(9) & 0 & 0 & -0.50(2) & -0.004(6) \\
0.045(2) & 0 & 0 & 0.70(3) & -0.005(6) \\
0.033(2) & -0.011(5) & 0.45(2) & 0 & 0 \\
0.044(5) & 0.003(5) & 0.27(1) & 0 & 0
\end{array}\right)
\end{aligned}
$$

C.4 Wilson action, $\beta=6.2, \mu^{2} a^{2}=0.617$

$$
\begin{array}{r}
\mathcal{Z}^{-}=\left(\begin{array}{rrrrr}
0.72(2) & 0 & 0 & 0 & 0 \\
0 & 0.68(1) & -0.31(1) & 0 & 0 \\
0 & -0.017(1) & 0.313(7) & 0 & 0 \\
0 & 0 & 0 & 0.286(8) & 0.001(3) \\
0 & 0 & 0 & 0.21(1) & 0.82(2)
\end{array}\right) \\
Z_{\chi}^{-}=\left(\begin{array}{rrrrr}
0.72(2) & 0 & 0 & 0 & 0 \\
0 & 0.69(1) & -0.29(1) & 0 & 0 \\
0 & -0.021(2) & 0.376(8) & 0 & 0 \\
0 & 0 & 0 & 0.291(9) & 0.002(3) \\
0 & 0 & 0 & 0.17(1) & 0.82(2)
\end{array}\right)
\end{array}
$$




$$
\begin{aligned}
\Delta^{-} & =\left(\begin{array}{rrrrr}
0 & -0.088(6) & 0.009(5) & -0.063(7) & -0.051(5) \\
-0.13(1) & 0 & 0 & 0.27(2) & 0.028(6) \\
-0.027(6) & 0 & 0 & 0.40(2) & 0.004(9) \\
-0.023(4) & -0.019(4) & 0.55(4) & 0 & 0 \\
-0.033(5) & 0.022(3) & -0.21(1) & 0 & 0
\end{array}\right) \\
\mathcal{Z}^{+} & =\left(\begin{array}{rrrrr}
0.60(1) & 0 & 0 & 0 & 0 \\
0 & 0.68(1) & 0.31(1) & 0 & 0 \\
0 & 0.017(2) & 0.312(7) & 0 & 0 \\
0 & 0 & 0 & 0.366(7) & -0.013(2) \\
0 & 0 & 0 & -0.287(9) & 0.78(1)
\end{array}\right) \\
Z_{\chi}^{+} & =\left(\begin{array}{rrrrr}
0.60(1) & 0 & 0 & 0 & 0 \\
0 & 0.68(1) & 0.27(1) & 0 & 0 \\
0 & 0.015(3) & 0.343(6) & 0 & 0 \\
0 & 0 & 0 & 0.411(7) & -0.014(2) \\
0 & 0 & 0 & -0.27(1) & 0.78(1)
\end{array}\right) \\
\Delta^{+} & \left(\begin{array}{rrrrr}
0 \\
0 & -0.155(9) & -0.012(5) & 0.050(6) & 0.024(5) \\
-0.118(7) & 0 & 0 & -0.33(2) & 0.006(6) \\
0.026(3) & 0 & 0 & 0.49(3) & -0.011(5) \\
0.015(2) & 0.004(5) & 0.33(2) & 0 & 0 \\
0.019(3) & 0.004(4) & 0.190(9) & 0 & 0
\end{array}\right)
\end{aligned}
$$

C.5 Clover action, $\beta=6.4, \mu^{2} a^{2}=0.313$

$$
\begin{aligned}
\mathcal{Z}^{-}= & \left(\begin{array}{rrrrr}
0.86(2) & 0 & 0 & 0 & 0 \\
0 & 1.00(2) & -0.50(2) & 0 & 0 \\
0 & -0.014(4) & 0.308(9) & 0 & 0 \\
0 & 0 & 0 & 0.229(9) & -0.035(6) \\
0 & 0 & 0 & 0.23(1) & 1.16(4)
\end{array}\right) \\
Z_{\chi}^{-} & \left(\begin{array}{rrrrr}
0.87(2) & 0 & 0 & 0 & 0 \\
0 & 1.04(2) & -0.51(2) & 0 & 0 \\
0 & -0.024(5) & 0.39(1) & 0 & 0 \\
0 & 0 & 0 & 0.27(1) & -0.042(7) \\
0 & 0 & 0 & 0.19(1) & 1.17(4)
\end{array}\right) \\
\Delta^{-} & =\left(\begin{array}{rrrrr}
0.116(9) & -0.02(2) & -0.13(2) & -0.11(2) \\
-0.17(1) & 0 & 0 & 0.31(1) & 0.02(3) \\
-0.044(7) & 0 & 0 & 0.45(2) & -0.04(2) \\
-0.05(1) & -0.04(1) & 0.71(4) & 0 & 0 \\
-0.08(1) & 0.029(8) & -0.24(1) & 0 & 0
\end{array}\right)
\end{aligned}
$$




$$
\begin{aligned}
\mathcal{Z}^{+} & =\left(\begin{array}{rrrrr}
0.92(1) & 0 & 0 & 0 & 0 \\
0 & 1.00(2) & 0.50(2) & 0 & 0 \\
0 & 0.015(2) & 0.31(1) & 0 & 0 \\
0 & 0 & 0 & 0.406(9) & -0.005(4) \\
0 & 0 & 0 & -0.54(2) & 1.37(4)
\end{array}\right) \\
Z_{\chi}^{+} & =\left(\begin{array}{rrrrr}
0.93(1) & 0 & 0 & 0 & 0 \\
0 & 1.00(2) & 0.42(2) & 0 & 0 \\
0 & 0.005(4) & 0.34(1) & 0 & 0 \\
0 & 0 & 0 & 0.47(1) & -0.005(5) \\
0 & 0 & 0 & -0.51(2) & 1.37(4)
\end{array}\right) \\
\Delta^{+} & =\left(\begin{array}{rrrrr}
0.01(2) & -0.01(1) & 0.12(2) & 0.04(1) \\
-0.18(1) & 0 & 0 & -0.43(3) & 0.002(19) \\
0.040(8) & 0 & 0 & 0.63(4) & 0.002(11) \\
0.027(7) & -0.012(4) & 0.39(2) & 0 & 0 \\
0.038(9) & 0.008(8) & 0.23(1) & 0 & 0
\end{array}\right)
\end{aligned}
$$

C.6 Wilson action, $\beta=6.4, \mu^{2} a^{2}=0.313$

$$
\begin{aligned}
\mathcal{Z}^{-} & =\left(\begin{array}{rrrrr}
0.68(1) & 0 & 0 & 0 & 0 \\
0 & 0.687(9) & -0.322(9) & 0 & 0 \\
0 & -0.014(2) & 0.275(5) & 0 & 0 \\
0 & 0 & 0 & 0.226(7) & -0.009(3) \\
0 & 0 & 0 & 0.192(9) & 0.82(3)
\end{array}\right) \\
Z_{\chi}^{-} & =\left(\begin{array}{rrrrr}
0.68(1) & 0 & 0 & 0 & 0 \\
0 & 0.697(8) & -0.32(1) & 0 & 0 \\
0 & -0.018(3) & 0.317(9) & 0 & 0 \\
0 & 0 & 0 & 0.242(7) & -0.010(4) \\
0 & 0 & 0 & 0.176(9) & 0.81(3)
\end{array}\right) \\
\Delta^{-} & =\left(\begin{array}{rrrrr}
0 & -0.084(4) & 0.012(9) & -0.06(1) & -0.04(1) \\
-0.123(6) & 0 & 0 & 0.251(8) & 0.02(1) \\
-0.034(3) & 0 & 0 & 0.36(1) & -0.007(9) \\
-0.033(5) & -0.025(7) & 0.54(3) & 0 & 0 \\
-0.025(8) & 0.014(5) & -0.182(8) & 0 & 0
\end{array}\right) \\
\mathcal{Z}^{+} & =\left(\begin{array}{rrrrr}
0.595(7) & 0 & 0 & 0 & 0 \\
0 & 0.686(9) & 0.321(8) & 0 & 0 \\
0 & 0.014(2) & 0.275(6) & 0 & 0 \\
0 & 0 & 0 & 0.335(5) & -0.011(2) \\
0 & 0 & 0 & -0.31(1) & 0.81(2)
\end{array}\right)
\end{aligned}
$$




$$
\begin{aligned}
& Z_{\chi}^{+}=\left(\begin{array}{rrrrr}
0.600(7) & 0 & 0 & 0 & 0 \\
0 & 0.683(9) & 0.299(9) & 0 & 0 \\
0 & 0.011(2) & 0.290(7) & 0 & 0 \\
0 & 0 & 0 & 0.363(7) & -0.012(2) \\
0 & 0 & 0 & -0.30(1) & 0.81(2)
\end{array}\right) \\
& \Delta^{+}=\left(\begin{array}{rrrrr}
0 & -0.142(9) & -0.012(5) & 0.061(7) & 0.015(6) \\
-0.111(5) & 0 & 0 & -0.30(2) & 0.003(10) \\
0.029(4) & 0 & 0 & 0.46(2) & -0.007(6) \\
0.015(2) & 0.002(3) & 0.30(1) & 0 & 0 \\
0.019(4) & 0.005(5) & 0.172(8) & 0 & 0
\end{array}\right)
\end{aligned}
$$

\section{References}

[1] G. Altarelli, G. Curci, G. Martinelli and S. Petrarca, Nucl. Phys. B187 (1981) 461

A.J. Buras and P.H. Weisz, Nucl. Phys. B333 (1990) 66.

[2] A.J. Buras, M. Jamin and P.H. Weisz, Nucl. Phys. B347 (1990) 491.

[3] A.J. Buras, M. Jamin M.E. Lautenbacher and P.H. Weisz, Nucl. Phys. B370 (1992) 69; Addendum Nucl. Phys. B375 (1992) 501; Nucl. Phys. B400 (1993) 37,75;

M. Ciuchini, E. Franco, G. Martinelli and L. Reina, Nucl. Phys. B415 (1994) 403; S. Herrlich and U. Nierste Nucl. Phys. B419 (1994) 292; Phys. Rev. D52 (1995) 6505; Nucl. Phys. B476 (1996) 27.

[4] M. Ciuchini et al., Z. Phys. C68 (1995) 239.

[5] M. Ciuchini et al., Nucl Phys. B523 (1998) 501.

[6] N. Cabibbo, G. Martinelli and R. Petronzio, Nucl. Phys. B244 (1984) 381;

R.C. Brower, M.B. Gavela, R. Gupta and G. Maturana, Phys. Rev. Lett. 53 (1984) 1318 ;

C. Bernard, in Argonne 1984, Proceedings of Gauge Theory on a Lattice, p. 85101, UCLA-84-TEP-03.

[7] B. Meyer and C. Smith, Phys. Lett. B123 (1983) 62;

G. Martinelli and Y.C. Zhang, Phys. Lett. B123 (1983) 433; Phys. Lett. B125 (1983) 77 ;

R. Groot, J. Hoek and J. Smit Nucl Phys. B237 (1984) 111;

E. Gabrielli et al., Nucl. Phys. B362 (1991) 475.

[8] G. Martinelli, Phys. Lett. B141 (1984) 395;

C. Bernard, A. Soni and T. Draper, Phys. Rev. D36 (1987) 3224.

[9] R. Frezzotti, E. Gabrielli, C. Pittori and G.C. Rossi, Nucl. Phys. B373 (1991) 781;

A. Borrelli, R. Frezzotti, E. Gabrielli and C. Pittori, Nucl. Phys. B409 (1993) 382.

[10] L.H. Karsten and J. Smit, Nucl. Phys. B183 (1981) 103. 
[11] M. Bochicchio et al., Nucl. Phys. B262 (1985) 331.

[12] L. Maiani and G. Martinelli, Phys. Lett. B178 (1986) 265.

[13] G. Martinelli and C.T. Sachrajda, Nucl. Phys. B306 (1988) 865; Nucl. Phys. B316 (1989) 305;

M. Crisafulli, G. Martinelli, V.J. Hill and C.T. Sachrajda, Phys. Lett. B223 (1989) 90;

G. Martinelli, C.T. Sachrajda and A. Vladikas, Nucl. Phys. B358 (1991) 212;

G. Martinelli, C.T. Sachrajda, G. Salina and A. Vladikas, Nucl. Phys. B378 (1992) 591; Nucl. Phys. B397 (1993) 479.

[14] G. Martinelli, S. Petrarca, C.T. Sachrajda and A. Vladikas, Phys. Lett. B311 (1993) 241; Phys. Lett. B317 (1993) 660.

[15] M. L. Paciello, S. Petrarca, B. Taglienti and A. Vladikas, Phys. Lett. B341 (1994) 187.

[16] UKQCD Collaboration, D.S. Henty, R.D. Kenway, B.J. Pendleton and J.I. Skullerud, Phys. Rev. D51 (1995) 5323.

[17] M. Crisafulli, V. Lubicz and A. Vladikas, Euro. Phys. J. C4 (1998) 145.

[18] JLQCD Collaboration, S. Aoki et al., Phys. Rev. Lett. 81 (1998) 1778; heplat/9901018.

[19] K. Jansen et al., Phys. Lett. B372 (1996) 275;

M. Lüscher, S. Sint, R. Sommer and P. Weisz, Nucl. Phys. B478 (1996) 365;

M. Lüscher, S. Sint, R. Sommer and H. Wittig, Nucl. Phys. B491 (1997) 344.

[20] G. Martinelli et al., Nucl. Phys. B445 (1995) 81.

[21] A. Donini et al., Phys. Lett. B360 (1996) 83.

[22] M. Göckeler et al., Nucl. Phys. B(Proc. Suppl.)47 (1996) 493; preprint DESY 98-097, hep-lat/9807044.

[23] A. Vladikas, Nucl. Phys. B(Proc. Suppl.)47 (1996) 84.

[24] A. Donini et al., Nucl. Phys. B(Proc. Suppl.)53 (1997) 883;

M Talevi, Nucl. Phys. B(Proc. Suppl.)60A (1998) 77.

[25] C. Dawson, G. Martinelli, G.C. Rossi, C.T. Sachrajda, S.R. Sharpe, M. Talevi and M. Testa, Nucl. Phys. B514 (1998) 313.

[26] G. Martinelli et al., Phys. Lett. B411 (1997) 141.

[27] M. Ciuchini et al., JHEP 10 (1998) 008.

[28] R. Gupta, T. Bhattacharya and S. Sharpe, Phys. Rev. D55 (1997) 4036.

[29] L. Conti et al., Phys. Let. B421 (1998) 273.

[30] C.R. Allton et al., preprint ROM2F/98/16, hep-lat/9806016. 
[31] N. Cabibbo, Phys. Rev. Lett. 12 (1964) 62; M. Gell-Mann, Phys. Rev. Lett. 12 (1964) 155.

[32] M. B. Gavela et al., Nucl. Phys. B306 (1988) 677; Nucl. Phys. B (Proc. Suppl.) 17 (1990) 769;

C. Bernard and A. Soni, Nucl. Phys. B (Proc. Suppl.) 17 (1990) 495; Nucl. Phys. B (Proc. Suppl.) 42 (1995) 391.

[33] R. Gupta et al., Phys. Rev. D47 (1993) 5113.

[34] T. Bhattacharya and R. Gupta, Nucl. Phys. B (Proc. Suppl.) 42 (1995) 935.

[35] S.R. Sharpe, Nucl. Phys. B (Proc. Suppl.) 34 (1994) 403;

N. Ishizuka et al., Nucl. Phys. B (Proc. Suppl.) 34 (1994) 403; Phys. Rev. Lett. 71 (1993) 24.

[36] S.R. Sharpe, Nucl. Phys. B (Proc. Suppl.) 53 (1997) 181.

[37] G.P. Lepage and P.B. Mackenzie, Phys. Rev. D48 (1993) 2250.

[38] B. Sheikholeslami and R. Wohlert, Nucl. Phys. B259 (1985) 572;

G. Heatlie et al., Nucl. Phys. B352 (1991) 266.

[39] M. Crisafulli et al., Phys. Lett. B369 (1996) 325.

[40] UKQCD Collaboration, L. Lellouch and C.-J. David Lin, hep-lat/9809148.

[41] C. Bernard, T. Draper, G. Hockney and A. Soni, Nucl. Phys. B (Proc. Suppl.) 4 (1988) 483.

[42] M. Testa, Nucl. Phys. B (Proc. Suppl.) 63 (1998) 38; JHEP 04 (1998) 002.

[43] C.R. Allton, V. Giménez, L. Giusti and F. Rapuano, Nucl. Phys. B489 (1997) 427;

V. Giménez, L. Giusti, F. Rapuano and M. Talevi, hep-lat/9801028. 

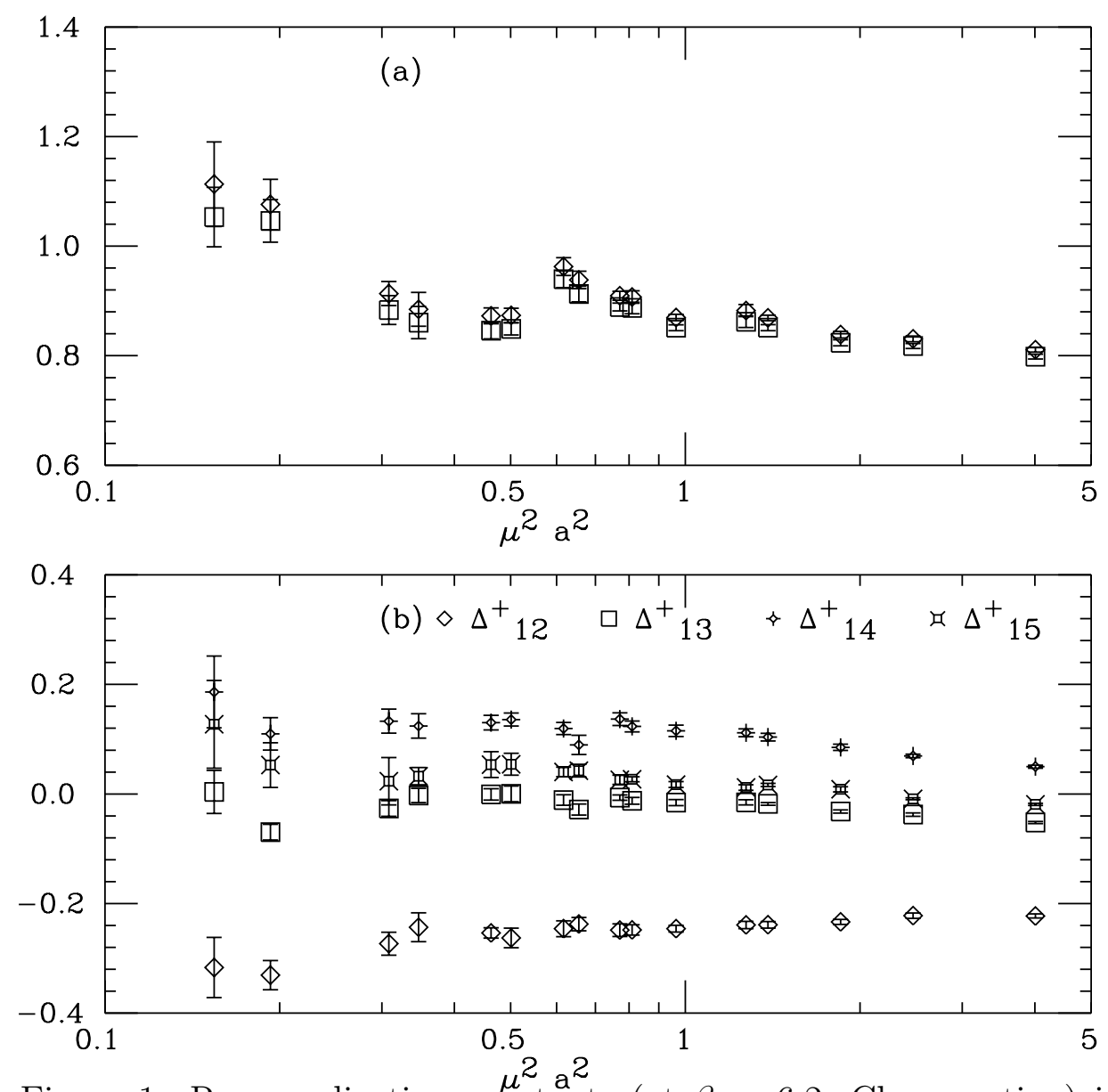

Figure 1: Renormalization constants (at $\beta=6.2$; Clover action) in the chiral limit as a function of the renormalization scale: (a) $Z_{11}^{+}(\diamond)$ and $\mathcal{Z}_{11}^{+}(\square)$; (b) $\Delta_{1 i}^{+}, i=2, \ldots, 5$. 

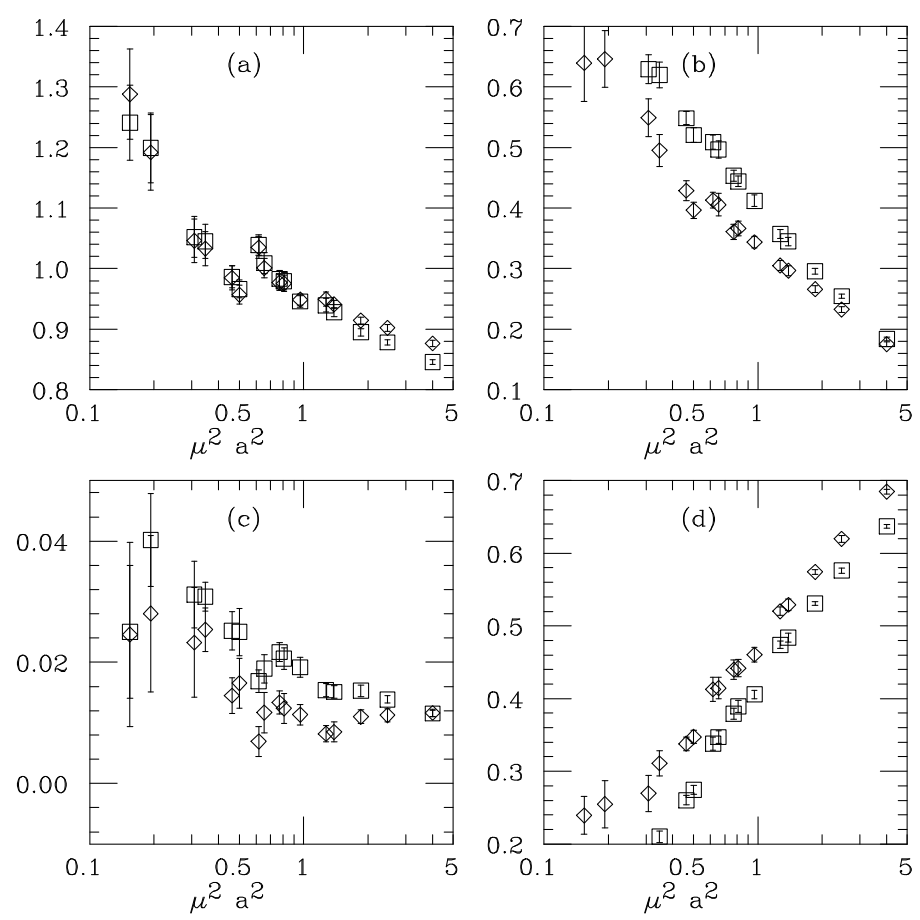

Figure 2: Renormalization constants (at $\beta=6.2$; Clover action) in the chiral limit as a function of the renormalization scale: (a) $Z_{22}^{+}(\diamond)$ and $\mathcal{Z}_{22}^{+}(\square)$; (b) $Z_{23}^{+}(\diamond)$ and $\mathcal{Z}_{23}^{+}(\square)$; (c) $Z_{32}^{+}(\diamond)$ and $\mathcal{Z}_{32}^{+}(\square) ;(\mathrm{d}) Z_{33}^{+}(\diamond)$ and $\mathcal{Z}_{33}^{+}(\square)$. 

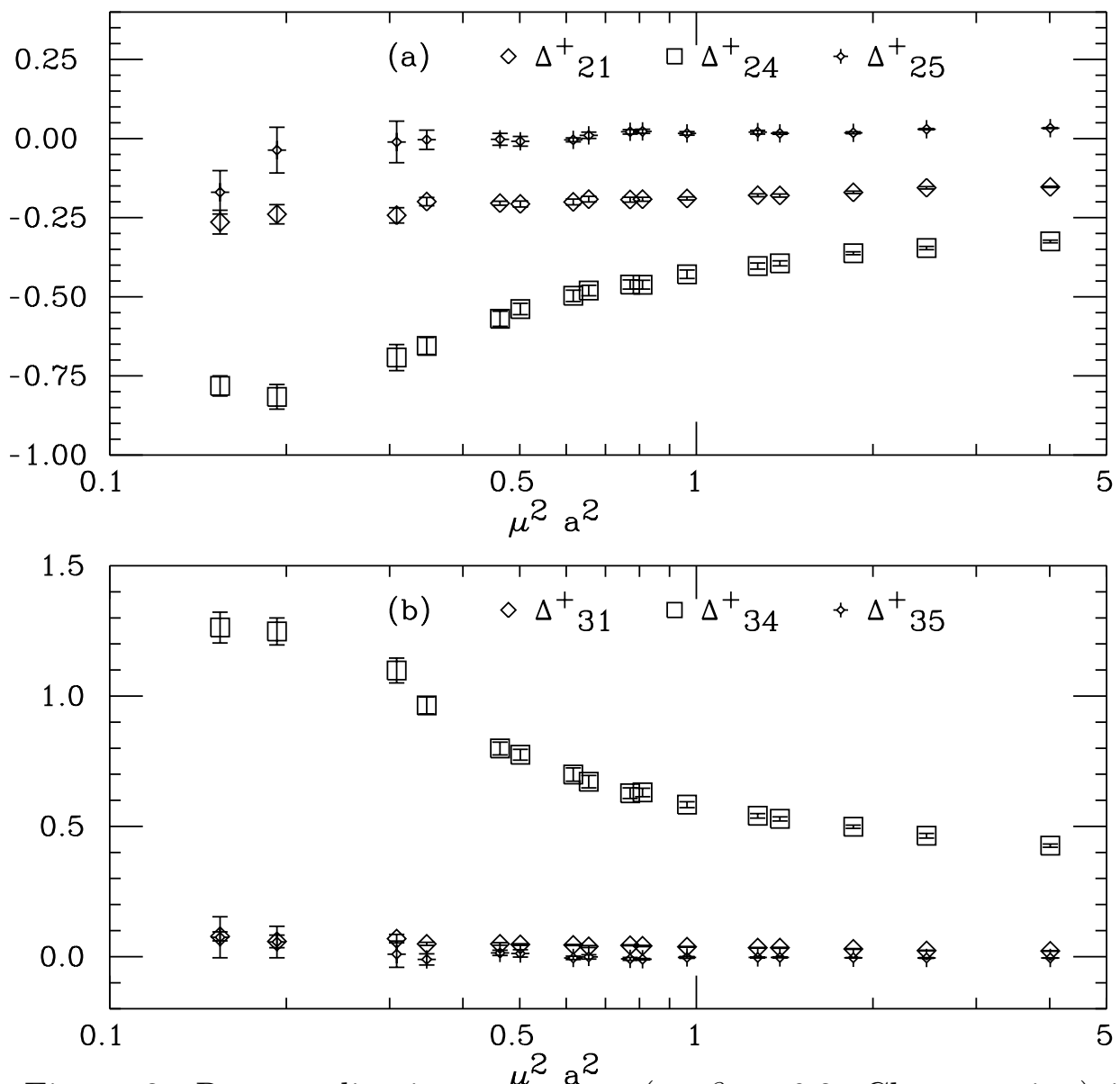

Figure 3: Renormalization constants (at $\beta=6.2$; Clover action) in the chiral limit as a function of the renormalization scale: (a) $\Delta_{2 i}^{+}, i=1,4,5$; (b) $\Delta_{3 i}^{+}, i=1,4,5$. 

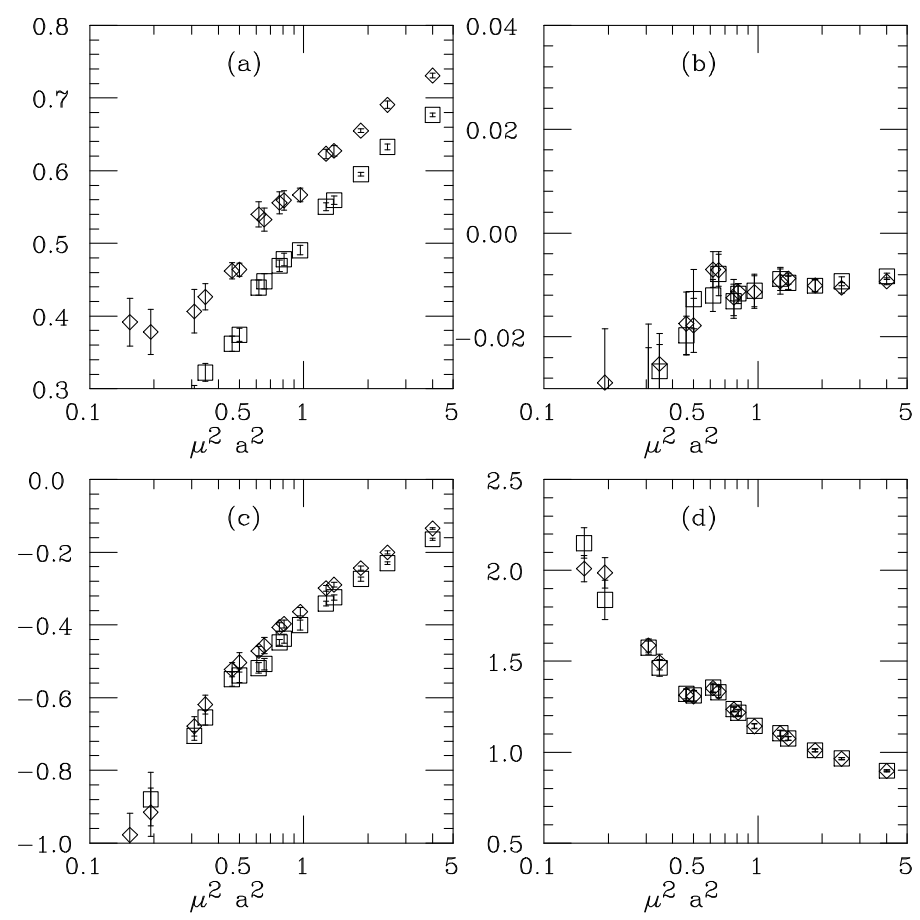

Figure 4: Renormalization constants (at $\beta=6.2$; Clover action) in the chiral limit as a function of the renormalization scale: (a) $Z_{44}^{+}(\diamond)$ and $\mathcal{Z}_{44}^{+}(\square)$; (b) $Z_{45}^{+}(\diamond)$ and $\mathcal{Z}_{45}^{+}(\square)$; (c) $Z_{54}^{+}(\diamond)$ and $\mathcal{Z}_{54}^{+}(\square) ;(\mathrm{d}) Z_{55}^{+}(\diamond)$ and $\mathcal{Z}_{55}^{+}(\square)$. 

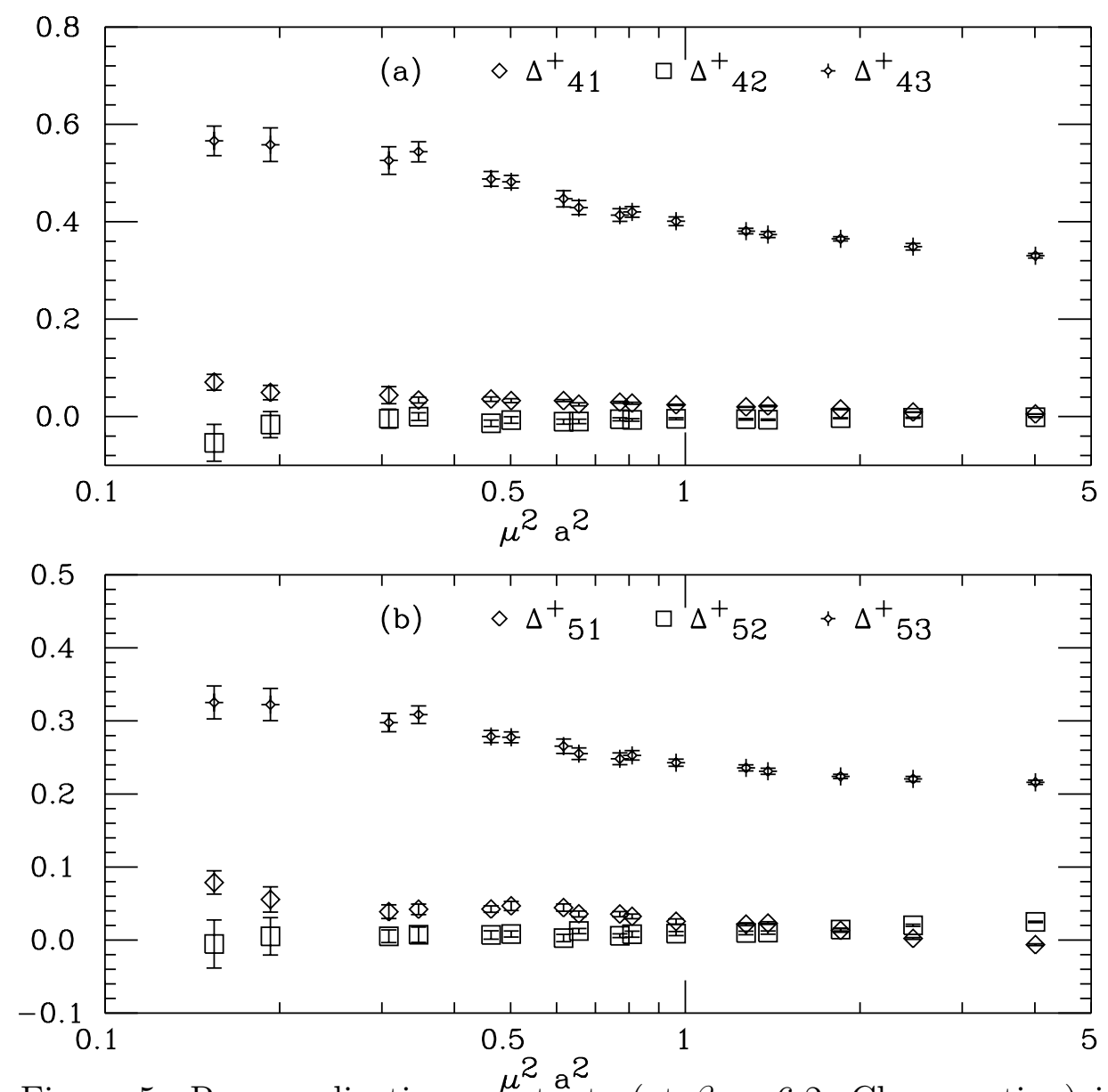

Figure 5: Renormalization constants (at $\beta=6.2$; Clover action) in the chiral limit as a function of the renormalization scale: (a) $\Delta_{4 i}^{+}, i=1,2,3$; (b) $\Delta_{5 i}^{+}, i=1,2,3$. 